\title{
Abundant betaines in giant clams (Tridacnidae) and western Pacific reef corals, including study of coral betaine acclimatization
}

\author{
Richard W. Hill ${ }^{1, *}$, Eric J. Armstrong ${ }^{1,3}$, Aaron M. Florn',4, Chao Li ${ }^{2,5}$, \\ Ryan W. Walquist ${ }^{1,6}$, Ahser Edward ${ }^{\mathrm{T}}$ \\ ${ }^{1}$ Department of Integrative Biology, Michigan State University, East Lansing, MI 48824, USA \\ ${ }^{2}$ Department of Chemistry, Michigan State University, East Lansing, MI 48824, USA \\ ${ }^{3}$ Present address: Department of Integrative Biology, University of California, Berkeley, CA 94720, USA \\ ${ }^{4}$ Present address: Nikon Instruments, Inc., 1300 Walt Whitman Road, Melville, NY 11747, USA \\ ${ }^{5}$ Present address: Takeda Pharmaceutical Company, 35 Landsdowne Street, Cambridge, MA 02139, USA \\ ${ }^{6}$ Present address: Department of Biological Sciences, Simon Fraser University, Vancouver, BC V6B 5K3, Canada
}

\begin{abstract}
A large literature documents that betaines play significant roles in protecting photosynthesis in the face of multiple stresses, including heat and photon stresses, in terrestrial plants and free-living algae. Betaines therefore can be expected to defend against photosystem stresses (e.g. photoinhibition and bleaching) in reef-building corals and tridacnid clams (both symbiotic with algae) in addition to functioning as osmolytes employed in osmotic stress defense. Nonetheless, the presence of betaines has just started to be studied in corals and has never before been investigated in tridacnids. The present research demonstrates the following. (1) Betaines, especially aminovaleric acid betaine and glycine betaine (GlyB), are abundant metabolites in all 4 major tissues of 5 tridacnid species studied. (2) Pacific corals have at least 9 betaines rather than only 1 as previously reported. (3) In regards to concentrations of betaines in Pacific corals, GlyB and proline betaine (ProB) typically dominate. Taxa differ in betaine profiles, however, including that Acropora spp. are exceptionally low in total betaines and Porites spp. have (in addition to GlyB and ProB) relatively high concentrations of alanine betaine, hydroxyproline betaine, and taurine betaine. (4) Genus-specific betaine profiles in corals may well be consistent across the Pacific basin. (5) During a year of laboratory acclimatization, coral species studied declined in bulk skeletal density and underwent both increases and decreases in betaine concentrations.
\end{abstract}

KEY WORDS: Stress resistance $\cdot$ Photoinhibition $\cdot$ Bleaching $\cdot$ Symbiodinium $\cdot$ Chemical ecology Metabolite - Osmolyte

\section{INTRODUCTION}

Betaines - amino (or imino) acids fully methylated at the $\mathrm{N}$ position - have recently been identified as abundant metabolites in the tissues of reef-building corals (Hill et al. 2010, Yancey et al. 2010). This discovery is important for several reasons, one being that betaines function as osmolytes in animals (Anthoni et al. 1991, de Vooys \& Geenevasen 2002, Yancey 2005, Yancey et al. 2010). Betaines also are

*Corresponding author: hillr@msu.edu

${ }^{ \pm}$Deceased; formerly at College of Micronesia-FSM, Kolonia, Pohnpei, Federated States of Micronesia

$\S_{\text {Corrections were made after publication. For details see }}$ www.int-res.com/abstracts/meps/v576/c_p27-41/

This corrected version: August 8, 2017 widely recognized as agents that stabilize proteins and membranes, helping to reduce cellular impacts of environmental stress (Rhodes \& Hanson 1993, McNeil et al. 1999).

Reef-building corals are under great stress at present. The corals are photosynthetic organisms, obligatorily symbiotic with algae termed zooxanthellaebelonging to the genus Symbiodinium - that are intracellular in the gastrodermis (Weis 2008). The algae carry out photosynthesis and perform vital

(c) The authors 2017. Open Access under Creative Commons by Attribution Licence. Use, distribution and reproduction are unrestricted. Authors and original publication must be credited.

Publisher: Inter-Research · www.int-res.com 
functions for the corals, including production of energy substrates (e.g. glucose) that are shared with the animal tissues in the symbiosis. One of the greatest threats to corals today is bleaching, a potentially lethal, stress-induced disruption of the symbiosis, marked by decline or loss of the algal symbionts (Hughes et al. 2003, Weis 2008). Bleaching principally results from synergistic actions of rising sea temperatures and photon stresses, with the primary insult being to the photosynthetic pathways in the zooxanthellae (Warner et al. 1999, Fitt et al. 2001, Jones \& Hoegh-Guldberg 2001, Lesser \& Farrell 2004, Weis 2008, Hill et al. 2010).

In terrestrial plants and free-living algae, betaines have been demonstrated by many studies to protect photosystem II (PSII) in the photosynthetic pathways against a number of abiotic stresses, including high temperature and high irradiance (Papageorgiou \& Murata 1995, Yang et al. 1996, Schiller \& Dau 2000, Allakhverdiev et al. 2003, 2008, Klimov et al. 2003, Hema et al. 2007, Li et al. 2014). The protective effects of betaines are sufficiently well established that multiple species of crop plants (or plants used as models in crop research) have been genetically engineered to increase betaine expression, as a way of enhancing crop resistance to photosystem stress imposed by environmental conditions (Alia et al. 1998, McNeil et al. 1999, Sakamoto \& Murata 2002, Prasad \& Saradhi 2004, Yang et al. 2007, Chen \& Murata 2011, Li et al. 2014). Betaines reduce photoinhibition in crop plants (Prasad \& Saradhi 2004, Yang et al. 2007, Li et al. 2014, Wang et al. 2014), and they increase defenses against reactive oxygen species in both crop plants and free-living algae (Prasad \& Saradhi 2004, Hema et al. 2007, Yang et al. 2007, Chen \& Murata 2011, Fan et al. 2012).

To understand the mechanisms of environmental stress effects on photosynthesis in corals - including photoinhibition and bleaching - investigators have made great progress by using prior studies on terrestrial plants and free-living algae as guides, as Lesser (2011) has emphasized. Coral betaines deserve study for this very reason. In view of the imminent threat posed by bleaching, the fact that betaines are well known as protective agents for photosynthesis in terrestrial plants suggests an imperative need to understand betaines in reef-building corals. Yet, until recently (Hill et al. 2010, Yancey et al. 2010), even the existence of betaines as abundant tissue metabolites in corals was conjectural at best. Hill et al. (2010) have provided preliminary ecological evidence that betaines play roles in photosystem stabilization in corals.
Whether betaines exist in the tissues of tridacnid (giant) clams has not been considered at all heretofore, although glycine betaine is known to be synthesized and accumulated by some other bivalve molluscs (Perrino \& Pierce 2000, de Vooys \& Geenevasen 2002). The tridacnid clams - which are important members of Indo-Pacific coral-reef communities - can be described in virtually identical terms to reefbuilding corals. They are photosynthetic because of being obligatorily symbiotic with Symbiodinium algae that, among other functions, make substantial contributions to their supplies of energy substrates (Klumpp \& Griffiths 1994, Hawkins \& Klumpp 1995). Moreover, tridacnid clams are susceptible to bleaching (Leggat et al. 2003). In the clams, the algal symbionts are extracellular, located in the tubular fluid of a profusely branching tubular system in the upwardfacing, siphonal mantle. Although the algae of tridacnids have traditionally been assigned to the same species as those of corals, Lee et al. (2015) have recently placed them in a distinct Symbiodinium species.

Recognizing that betaines may play important protein- and membrane-stabilizing roles or osmolyte roles, we had 3 principal goals for the present research. First, we wanted to initiate the study of betaines in tridacnid clams by carrying out a chemically definitive study of the types and concentrations of betaines in tridacnid tissues. Second, we sought to expand knowledge of betaines in Pacific reef-building corals by examining additional populations and species, and by quantifying betaines not heretofore measured in Pacific corals. A particular interest in our studies of corals was to determine if widespread coral species are consistent in their betaine profiles in the western and eastern Pacific and to expand sampling within coral genera to learn more about the apparent tendency of some genera to exhibit consistently different betaine profiles from others. Third, we wanted to study metabolite acclimatization. Specifically, we investigated betaine concentrations - and skeletal density - as functions of time during a year of laboratory acclimatization in 2 coral species. There have been few prior studies of metabolite phenotypic plasticity in corals, and none of betaine plasticity.

\section{MATERIALS AND METHODS}

\section{Specimens}

Animals were collected at Pohnpei, Federated States of Micronesia (corals and clams) $\left(6.96^{\circ} \mathrm{N}, 158.2^{\circ} \mathrm{E}\right)$, and at the Republic of Palau (clams) $\left(7.34^{\circ} \mathrm{N}, 134.5^{\circ} \mathrm{E}\right)$. 
Wild corals were collected at depths of 1-10 m on the outer fringing reef offshore from Kolonia, Pohnpei. Species obtained were Acropora formosa, Montipora monasteriata, Pavona varians, Pocillopora damicornis, Porites cylindrica, and Porites massive (we follow Turak \& DeVantier 2005 in using 'Porites massive' to refer to specimens of Porites lobata or Porites lutea, which could not be distinguished under our collecting conditions). Species with branching growth forms were sampled by cutting off short segments of lightexposed, outer branches, whereas those with massive growth forms were sampled by removing light-exposed, upward-facing surfaces with chisel and hammer. Each coral specimen studied was from a different colony and placed immediately in an individual plastic bag with local seawater. Juvenile clams - Tridacna crocea (shell length: 3.7-4.9 cm), T. derasa $(7.4-9.1 \mathrm{~cm})$, and T. maxima $(4.8-5.9 \mathrm{~cm})$ - were obtained from the Palau Mariculture Demonstration Center, Koror, Palau. They had been reared from an early age in open-topped, unshaded, outdoor sea tables provided with flowing natural seawater (water depth: ca. $30 \mathrm{~cm}$ ). Wild adult Hippopus hippopus (long axis measuring $14.8-20.9 \mathrm{~cm}$ ) were collected at 2-3 m depth, and wild adult $T$. maxima $(8.5-17.5 \mathrm{~cm})$ and $T$. squamosa $(13.7 \mathrm{~cm})$ were collected at 1-10 $\mathrm{m}$ depth on Kolonia reefs. Wild adult T. crocea $(6.7-10.2 \mathrm{~cm})$ were collected at depths averaging $1.3 \mathrm{~m}$. In the field, all coral and clam specimens were promptly placed in an insulated box that prevented light exposure and warming.

After return to a lab, coral pieces adequate to provide $3-15 \mathrm{~cm}^{2}$ of living polyp tissue were placed in $15 \mathrm{ml}$ Teflon vials (Nalgene) and immediately frozen at $-20^{\circ} \mathrm{C}$. From each adult clam, we cut four 1 to $2 \mathrm{~g}$ tissue samples (similar to the sampling in R. W. Hill et al. 2004): (1) light-exposed, siphonal mantle, (2) shaded mantle from near the byssal opening, (3) adductor muscle, and (4) gill (including both right and left). Siphonal mantle was sampled from each immature clam, but the other 3 tissues of immatures were sometimes too small to be useful or not sampled because of limits on cryogenic transport. Clam tissue samples were placed in Corning 1.2 or $2.0 \mathrm{ml}$ polypropylene cryogenic vials and frozen at $-20^{\circ} \mathrm{C}$.

Zooxanthellae were isolated from 12 adult clams (2 H. hippopus, 9 T. maxima, and 1 T. squamosa) by the procedure of Jeffrey \& Haxo (1968). For sampling each clam, 7-19 $\mathrm{g}$ of siphonal mantle were diced with scissors, placed in $100 \mathrm{ml}$ of clear, local reef seawater that had been filtered through a Pall Gelman A/E glass fiber filter, and pulverized in a Hamilton Beach 51101 blender for $1 \mathrm{~min}$. The slurry was filtered by gravity through cheese cloth (6-8 layers). A $60 \mathrm{ml}$ subsample of the filtrate was centrifuged using a standard clinical centrifuge for $1.5 \mathrm{~min}$, after which the pellet was put through 2 cycles of being resuspended in fresh filtered seawater and re-centrifuged. After the final pellet was resuspended in fresh filtered seawater, the suspension was passed by gravity through an A/E glass fiber filter, and the filter (with zooxanthellae) was frozen in a Corning cryogenic vial.

The total number of coral and clam tissue specimens was limited to 260 by constraints on cryogenic transport. Authorizing permits for collection and/or export were Export Permit PW08-002, Bureau of Marine Resources, Republic of Palau; Specimen Collection Permit MD001-09, Marine Development Office of Economic Affairs, Pohnpei State Government; and Certificate of Verification PNI001A (January 10, 2009), Department of Resources and Development, Federated States of Micronesia.

Samples (refrigerated in transit with Pelton-Shepherd R-type plastic-ice frozen at $-20^{\circ} \mathrm{C}$ ) were flown to Guam 1-9 d after clam collection (depending on collection date) and 1-4 d after coral collection. Samples were transferred to dry ice in Guam and maintained in dry ice for $46 \mathrm{~h}$, until arrival at Michigan State University (MSU), where they were stored at $-80^{\circ} \mathrm{C}$ until analyzed.

\section{Study of coral acclimatization}

Fragments (3-5 cm high) of living Acropora cerealis and Montipora digitata glued to plastic or plaster bases were obtained from the Marine and Environmental Research Institute of Pohnpei (MERIP). These had been propagated by serial fragmentation in a mariculture setting in the Northeast Pohnpei Lagoon at depths of about $1 \mathrm{~m}$ (M. digitata) or $5 \mathrm{~m}$ (A. cerealis). Each species had originated in the past with multiple wild-collected specimens from Pohnpei reefs. Six fragments of each species were collected simultaneously with the wild corals, processed in Pohnpei in the same way as wild corals, and transported to MSU frozen under authority of the aforementioned permits (see previous subsection).

Three weeks after collection of these 12 fragments, MERIP shipped 25 living fragments of each species to MSU (CITES permit 1-19-09-01, Department of Resources and Development, Federated States of Micronesia; US Fish and Wildlife Service Declaration for Importation of Fish or Wildlife, Import License 797686). The 50 living fragments arrived 1 calendar 
day after shipment and were placed in a sea table with rapidly flowing, well-conditioned artificial seawater (Tropic Marin) used routinely for coral aquaculture at A\&M Aquatics, Lansing, MI. Coral samples for analysis (quick-frozen at $-80^{\circ} \mathrm{C}$, averaging $6.2 \mathrm{~cm}^{2}$ surface area, range: $2.8-12.9 \mathrm{~cm}^{2}$ ) were clipped periodically from new growth of these 50 living coral fragments over the ensuing 13 mo. Our goal was to obtain 6 clippings from each species on each sampling date. In the sea table, water temperature averaged $25^{\circ} \mathrm{C}$. Light was provided $10 \mathrm{~h}$ each day from overhead $10000 \mathrm{~K}$ metal halide lamps at an intensity, at the water surface, averaging $190 \mu \mathrm{mol}$ einstein $\mathrm{s}^{-1} \mathrm{~m}^{-2}$, with the corals at an average depth of $10.5 \mathrm{~cm}$. Water quality characteristics (salinity, alkalinity, major ion concentrations), checked every $3 \mathrm{~d}$, were stable.

The bulk density of the skeleton (Bucher et al.1998) in all samples in the acclimatization study was measured, in addition to measurement of metabolites. After the entire surface of each sample had been blasted to remove tissue (see next subsection), the sample was dried ( $\geq 2 \mathrm{wk}$ ) in room air, then dried for $24 \mathrm{~h}$ at $60^{\circ} \mathrm{C}$ prior to measurement of dry weight. The surface of the sample was then sealed by dipping in molten Paraplast (Lancer), and the sample was weighed while submerged in distilled water. Bulk density was calculated as in Bucher et al. (1998), correcting their equation for total volume so that we divided by water density rather than multiplying by it.

\section{Chemical analysis of metabolites and chlorophyll}

Chemical methods were based on extensive validation studies not detailed here. Each clam tissue sample was powdered in liquid nitrogen by mortar and pestle. Powder weighing ca. $0.1 \mathrm{~g}$ was then mixed with $1.5 \mathrm{ml}$ of extraction solution $(48.5 \%$ methanol, $48.5 \%$ distilled [Milli-Q] water, $3 \%$ formic acid). The mixture was vortexed $(10 \mathrm{~s})$, sonicated on ice (10 min), centrifuged at $4^{\circ} \mathrm{C}(15000 \times g$ for $10 \mathrm{~min})$, and the last 3 steps repeated. The supernatant was then removed, and the pellet was resuspended in $1.5 \mathrm{ml}$ of fresh extraction solution, vortexed, sonicated, and centrifuged, after which the supernatant was removed and combined with the earlier supernatant, forming the mixed supernatant. Internal standards were immediately added to a subsample, which was then evaporated dry at room temperature or below (SpeedVac).

To prepare a coral specimen for analysis, the methods of Hill et al. (2010) were followed. After blasting an area measuring 3-15 $\mathrm{cm}^{2}$ with ice-cold distilled water, blastate was immediately mixed 2:1 with methanol and acidified to $3 \%$ formic acid. Internal standards were added to a subsample, which was sonicated on ice $(10 \mathrm{~min})$, centrifuged at $4^{\circ} \mathrm{C}(8000$ $\times g$ for $5 \mathrm{~min}$ ), vortexed to resuspend the pellet, and centrifuged again. A portion of supernatant was then evaporated dry (SpeedVac).

Following evaporation, the evaporate of a clam or coral sample was dissolved in mobile phase, consisting of water (Milli-Q) adjusted by addition of formic acid to $\mathrm{pH} 3.85$ - the $\mathrm{pH}$ that optimized chromatographic resolution of isobaric and isomeric zwitterionic metabolites. Analysis of this solution was then executed using liquid chromatography and timeof-flight mass spectrometry (LC-MS) employing a Supelco Discovery-HS F5 pentafluorophenylpropyl HPLC column and Shimadzu LC-20AD HPLC pump, coupled to a Waters LCT Premier mass spectrophotometer operated using electrospray ionization in positive mode (Li et al. 2010).

Quantification of 7 betaines-alanine betaine (AlaB), aminovaleric acid betaine (AValB), $\beta$-alanine betaine $(\beta \mathrm{AlaB}), \gamma$-aminobutyric acid betaine (GA$\mathrm{BAB})$, glycine betaine (GlyB), hydroxyproline betaine (HProB), and proline betaine (ProB) - was carried out using matching deuterated internal standards $\left(\mathrm{d}_{9}\right.$ AlaB, d $d_{9}-A V a l B, d_{9}-\beta A l a B, d_{9}-G A B A B, d_{9}-G_{y} B, d_{6}-$ HProB, and $d_{6}$-ProB) that had been synthesized from amino acids and $\mathrm{CD}_{3} \mathrm{I}$ (Chen \& Benoiton 1976, Hill et al. 2010). The purities and concentrations of all standards were established using proton nuclear magnetic resonance $\left({ }^{1} \mathrm{H}-\mathrm{NMR}\right)$ and LC-MS. A compound identified from exact mass and molecular fragmentation studies as taurine betaine (TauB) was often observed, as was a compound identified as trigonelline (Trig) and/or homarine (Hom), betaines that cannot be distinguished with our methods. These compounds were quantified against $d_{9}$-GlyB (Hill et al. 2010).

Tissue dimethylsulfoniopropionate (DMSP) was quantified in a subset of clam samples $(\mathrm{n}=24)$ using DMSP purchased from Research Plus (Barnegat, NJ) as an external standard. The DMSP was added to a subsample of the mixed supernatant, which was evaporated and then resuspended in mobile phase for LC-MS. In the coral acclimatization study, deuterated DMSP $\left(d_{6}-D M S P\right.$; see Hill et al. 2010 for synthesis) was added at a consistent concentration to all coral samples at the time of addition of the betaine internal standards. This DMSP permitted relative changes in the native DMSP concentration to be measured.

The concentration of photopigment was measured in each clam tissue and coral blastate. To this end, a light-protected subsample of clam tissue powder was mixed with cold $90 \%$ acetone, or a light-protected 
subsample of coral blastate was mixed with 9 times its volume of pure, cold acetone, creating a $90 \%$ acetone solution. After 3-4 h of extraction in darkness, solids were removed by centrifugation. Chlorophyll in the supernatant was quantified with a Turner TD700 fluorometer calibrated with standards of spinach chlorophyll a (Sigma-Aldrich). Thereafter the supernatant was acidified by addition of $\mathrm{HCl}$ to a concentration of $3 \mathrm{mM}$ to convert all chlorophyll to pheophytin, and pheophytin was quantified with acidified standards. Results are reported as pheophytin to remove potential effects of variable degradation of chlorophyll to pheophytin prior to analysis during specimen storage and transport.

\section{Measurement of coral surface area}

Coral metabolite data were normalized to the area of polyp tissue removed by blasting, area being measured on the skeleton remaining after blasting. For Porites cylindrica and Montipora digitata, both of which have simple growth forms, the area blasted (3$13 \mathrm{~cm}^{2}$ ) was measured by digital image processing (Scion Image) using images of thin aluminum foil previously fitted to coral contours. For all other corals, the area blasted $\left(3-15 \mathrm{~cm}^{2}\right)$ was measured by double wax dipping, found to be superior for Acropora spp. by Naumann et al. (2009). We used Paraplast (Lancer; melting point: $56-57^{\circ} \mathrm{C}$ ) at $60^{\circ} \mathrm{C}$, and we calibrated with wooden calibration objects that spanned the full range of coral areas measured. During measurement by wax dipping, surfaces that had not been covered with polyps, such as breaks caused by clipping, were measured with digital image processing and their areas subtracted from the total area measured with wax.

\section{Statistics}

Statistical calculations were carried out in IBM SPSS 19.0 for Windows. Normality and homoscedasticity were assessed by probit plots and Levene's test. Null hypotheses were rejected if the likelihood of the results obtained was $<0.05$ under the null hypothesis.

\section{RESULTS}

\section{Extraction efficiency}

The extraction efficiency of the coral extraction method was previously demonstrated to exceed $99 \%$ for AlaB, BAlaB, DMSP, GlyB, HProB, ProB, TauB, and Trig/Hom (Hill et al. 2010). To measure the extraction efficiency of the clam extraction method, we studied 18 adult clam tissue samples —including all 4 tissue types - by extracting them an extra time following the initial extraction as described in 'Materials and methods'. Extraction efficiency was calculated by dividing the amount in the initial extraction by the total in both extractions. In 17 tissue samples, the average extraction efficiency for all betaines except TauB was over $98 \%$. However, 1 sample exhibited lower extraction (Table 1). Two compounds, TauB and HProB, were distinctive. Because TauB extraction efficiency was erratic (in the 17 samples: $65.3 \pm 6.9 \%$ ), detailed clam TauB data are not reported. HProB was undetectable in 5 of the 18 samples. In the remaining 13, however, HProB extraction efficiency was high $(98.5 \pm 0.85 \%)$, except in the outlier sample (Table 1), where it was $70.8 \%$.

\section{Betaines in tridacnid tissues}

Concentrations of the 6 most abundant betaines in clam tissues are summarized in Tables $2 \& 3$. Besides these 6 betaines, AlaB and GABAB were detected in almost all species and tissues, but their concentrations were nearly always $<1 \mu \mathrm{mol} \mathrm{g}^{-1}$ (except that in adductor samples, AlaB averaged $1.5 \mathrm{\mu mol} \mathrm{g}^{-1}$ in adult Hippopus hippopus, and GABAB averaged 1.2-2.8 $\mu \mathrm{mol}$ $\mathrm{g}^{-1}$ in adult Tridacna maxima and T. crocea). TauB

Table 1. Percentage of betaines and DMSP extracted by the standard extraction method described in 'Materials and methods, chemical analysis of metabolites and chlorophyll' in 18 adult Tridacna maxima tissue samples (4 of adductor muscle, 4 of byssal mantle, 5 of gill, and 5 of siphonal mantle) that were extracted an extra time following the standard extraction. One adductor sample is presented separately because of extraction efficiences distinctly different from those seen in the other 17 samples. AlaB: alanine betaine, AValB: aminovaleric acid betaine, $\beta$ AlaB: $\beta$-alanine betaine, GABAB: $\gamma$-aminobutyric acid betaine, GlyB: glycine betaine, ProB: proline betaine, Trig/Hom: trigonelline and/or homarine

\begin{tabular}{|lcc|}
\hline Compound & $\begin{array}{c}\text { 17 samples } \\
\text { (mean } \pm \text { SE, range) }(\%)\end{array}$ & $\begin{array}{c}\text { 1 adductor } \\
\text { sample (\%) }\end{array}$ \\
\hline AlaB & $98.8 \pm 0.21(97.2-99.8)$ & 92.5 \\
AValB & $98.2 \pm 0.28(94.8-99.3)$ & 73.1 \\
BAlaB & $99.0 \pm 0.21(97.3-99.9)$ & 75.5 \\
DMSP & $98.6 \pm 0.20(96.6-99.5)$ & 69.9 \\
GABAB & $99.1 \pm 0.27(95.0-99.8)$ & 77.3 \\
GlyB & $98.2 \pm 0.22(96.1-99.3)$ & 77.7 \\
ProB & $98.7 \pm 0.55(90.4-99.9)$ & 78.8 \\
Trig/Hom & $99.1 \pm 0.13(98.1-99.8)$ & 80.6 \\
\hline
\end{tabular}


Table 2. Mean \pm SE (range) tissue concentrations of the 6 most abundant betaines and pheophytin in tissues of adult tridacnid clams of 4 species. HProB: hydroxyproline betaine. See Table 1 for definitions of other chemical abbreviations. n: number of independently collected individuals. nd: not measured because of a flaw in analysis

\begin{tabular}{|c|c|c|c|c|c|c|c|}
\hline Tissue & $\begin{array}{c}\text { AValB } \\
\left(\mu \mathrm{mol} \mathrm{g}{ }^{-1}\right)\end{array}$ & $\underset{\left(\mu \mathrm{molaB} \mathrm{g}^{-1}\right)}{\alpha}$ & $\begin{array}{c}\text { GlyB } \\
\left(\mu \mathrm{mol} \mathrm{g}{ }^{-1}\right)\end{array}$ & $\begin{array}{c}\text { HProB } \\
\left(\mu \mathrm{mol} \mathrm{g}{ }^{-1}\right)\end{array}$ & $\begin{array}{c}\text { ProB } \\
\left(\mu \mathrm{mol} \mathrm{g}{ }^{-1}\right)\end{array}$ & $\begin{array}{l}\text { Trig/Hom } \\
\left(\mu \mathrm{mol} \mathrm{g}{ }^{-1}\right)\end{array}$ & $\begin{array}{c}\text { Pheophytin } \\
\left(\mu g g^{-1}\right)\end{array}$ \\
\hline \multicolumn{8}{|c|}{ Tridacna maxima $(\mathrm{n}=16)$} \\
\hline Adductor & $\begin{array}{c}67 \pm 17 \\
(11-295)\end{array}$ & $\begin{array}{c}0.20 \pm 0.027 \\
(0.04-0.39)\end{array}$ & $\begin{array}{l}129 \pm 13 \\
(59-240)\end{array}$ & $\begin{array}{c}2.1 \pm 0.59 \\
(0-9.4)\end{array}$ & $\begin{array}{l}2.1 \pm 0.28 \\
(0.50-4.7)\end{array}$ & $\begin{array}{c}57 \pm 9.7 \\
(15-160)\end{array}$ & $\begin{array}{c}0.094 \pm 0.031 \\
(0-0.39)\end{array}$ \\
\hline Byssal mantle & $\begin{array}{l}8.1 \pm 1.2 \\
(2.6-23)\end{array}$ & $\begin{array}{l}2.0 \pm 0.56 \\
(0.46-9.6)\end{array}$ & $\begin{array}{l}16 \pm 2.2 \\
(5.2-37)\end{array}$ & $\begin{array}{c}0.93 \pm 0.30 \\
(0.01-4.8)\end{array}$ & $\begin{array}{c}0.70 \pm 0.13 \\
(0.18-2.1)\end{array}$ & $\begin{array}{l}6.6 \pm 1.1 \\
(2.4-15)\end{array}$ & $\begin{array}{c}0.47 \pm 0.075 \\
(0.06-1.3)\end{array}$ \\
\hline Gill & $\begin{array}{c}0.96 \pm 0.15 \\
(0.30-2.4)\end{array}$ & $\begin{array}{c}3.7 \pm 0.82 \\
(1.0-14)\end{array}$ & $\begin{array}{c}48 \pm 6.1 \\
(18-103)\end{array}$ & $\begin{array}{c}0.076 \pm 0.021 \\
(0-0.40)\end{array}$ & $\begin{array}{c}0.73 \pm 0.12 \\
(0.18-1.8)\end{array}$ & $\begin{array}{l}10 \pm 1.7 \\
(4.5-33)\end{array}$ & $\begin{array}{l}2.3 \pm 0.52 \\
(0.10-7.8)\end{array}$ \\
\hline Siphonal mantle & $\begin{array}{l}19 \pm 2.2 \\
(8.3-47)\end{array}$ & $\begin{array}{l}5.1 \pm 1.5 \\
(1.3-21)\end{array}$ & $\begin{array}{c}26 \pm 6.0 \\
(7.5-109)\end{array}$ & $\begin{array}{c}0.61 \pm 0.24 \\
(0-3.8)\end{array}$ & $\begin{array}{c}0.92 \pm 0.19 \\
(0.23-2.5)\end{array}$ & $\begin{array}{l}8.8 \pm 3.3 \\
(2.1-56)\end{array}$ & $\begin{array}{l}207 \pm 22 \\
(71-387)\end{array}$ \\
\hline \multicolumn{8}{|c|}{ T. squamosa $(\mathrm{n}=1)$} \\
\hline Adductor & 1.9 & 0.11 & 107 & 0.29 & 0.59 & nd & 0 \\
\hline Byssal mantle & 2.3 & 0.81 & 13 & 0.3 & 0.38 & 3.0 & 0.14 \\
\hline Gill & 0.21 & 1.7 & 29 & 0.015 & 0.40 & 4.0 & 0.62 \\
\hline Siphonal mantle & 9.5 & 2.9 & 18 & 0.18 & 0.50 & 5.0 & 176 \\
\hline \multicolumn{8}{|l|}{ T. $\operatorname{crocea}(\mathrm{n}=10)$} \\
\hline Adductor & $\begin{array}{c}57 \pm 9.9 \\
(12-128)\end{array}$ & $\begin{array}{c}0.35 \pm 0.059 \\
(0.14-0.82)\end{array}$ & $\begin{array}{l}79 \pm 5.5 \\
(51-109)\end{array}$ & $\begin{array}{l}2.8 \pm 0.61 \\
(0.78-7.2)\end{array}$ & $\begin{array}{l}1.7 \pm 0.21 \\
(0.51-2.9)\end{array}$ & $\begin{array}{l}34 \pm 3.5 \\
(9.0-46)\end{array}$ & $\begin{array}{c}0.048 \pm 0.018 \\
(0.00-0.13)\end{array}$ \\
\hline Byssal mantle & $\begin{array}{l}22 \pm 5.3 \\
(5.6-59)\end{array}$ & $\begin{array}{c}6.1 \pm 0.65 \\
(4.1-9.5)\end{array}$ & $\begin{array}{l}16 \pm 1.7 \\
(9.9-26)\end{array}$ & $\begin{array}{l}1.6 \pm 0.31 \\
(0.86-3.9)\end{array}$ & $\begin{array}{l}1.4 \pm 0.27 \\
(0.34-2.4)\end{array}$ & $\begin{array}{c}5.6 \pm 0.78 \\
(3.5-9.1)\end{array}$ & $\begin{array}{l}0.24 \pm 0.062 \\
(0.060-0.54)\end{array}$ \\
\hline Gill & $\begin{array}{l}2.9 \pm 0.48 \\
(0.72-5.0)\end{array}$ & $\begin{array}{c}2.5 \pm 0.21 \\
(1.7-3.3)\end{array}$ & $\begin{array}{l}40 \pm 2.7 \\
(29-51)\end{array}$ & $\begin{array}{c}0.29 \pm 0.043 \\
(0.20-0.52)\end{array}$ & $\begin{array}{l}1.2 \pm 0.12 \\
(0.62-1.6)\end{array}$ & $\begin{array}{c}13 \pm 0.69 \\
(8.8-14)\end{array}$ & $\begin{array}{c}0.26 \pm 0.024 \\
(0.18-0.34)\end{array}$ \\
\hline Siphonal mantle & $\begin{array}{l}25 \pm 3.7 \\
(13-48)\end{array}$ & $\begin{array}{c}10 \pm 0.74 \\
(5.8-14)\end{array}$ & $\begin{array}{l}18 \pm 3.2 \\
(6.2-32)\end{array}$ & $\begin{array}{c}0.54 \pm 0.14 \\
(0-1.2)\end{array}$ & $\begin{array}{l}1.3 \pm 0.19 \\
(0.62-2.5)\end{array}$ & $\begin{array}{l}7.8 \pm 1.2 \\
(4.1-14)\end{array}$ & $\begin{array}{c}245 \pm 28 \\
(161-429)\end{array}$ \\
\hline \multicolumn{8}{|c|}{ Hippopus hippopus $(\mathrm{n}=4)$} \\
\hline Adductor & $\begin{array}{c}63 \pm 19 \\
(35-118)\end{array}$ & $\begin{array}{c}2.7 \pm 2.2 \\
(0.31-9.2)\end{array}$ & $\begin{array}{l}71 \pm 11 \\
(50-97)\end{array}$ & $\begin{array}{c}0.83 \pm 0.075 \\
(0.67-1.0)\end{array}$ & $\begin{array}{l}6.7 \pm 1.5 \\
(3.9-9.9)\end{array}$ & $\begin{array}{l}35 \pm 6.1 \\
(25-51)\end{array}$ & $\begin{array}{c}0.020 \pm 0.012 \\
(0.00-0.042)\end{array}$ \\
\hline Byssal mantle & $\begin{array}{c}5.2 \pm 2.4 \\
(0.82-9.0)\end{array}$ & $\begin{array}{c}0.84 \pm 0.46 \\
(0.29-1.8)\end{array}$ & $\begin{array}{l}36 \pm 8.6 \\
(19-47)\end{array}$ & $\begin{array}{l}0.049 \pm 0.011 \\
(0.027-0.063)\end{array}$ & $\begin{array}{c}0.24 \pm 0.0090 \\
(0.23-0.26)\end{array}$ & $\begin{array}{c}7.5 \pm 0.59 \\
(6.8-8.6)\end{array}$ & $\begin{array}{l}5.6 \pm 2.9 \\
(0.15-10)\end{array}$ \\
\hline Gill & $\begin{array}{l}1.5 \pm 0.40 \\
(0.64-2.5)\end{array}$ & $\begin{array}{l}1.6 \pm 0.46 \\
(0.72-2.8)\end{array}$ & $\begin{array}{l}54 \pm 7.0 \\
(44-74)\end{array}$ & $\begin{array}{c}0.18 \pm 0.026 \\
(0.11-0.23)\end{array}$ & $\begin{array}{c}0.85 \pm 0.13 \\
(0.51-1.1)\end{array}$ & $\begin{array}{l}11 \pm 1.3 \\
(9.1-16)\end{array}$ & $\begin{array}{c}0.048 \pm 0.028 \\
(0.00-0.10)\end{array}$ \\
\hline Siphonal mantle & $\begin{array}{l}5.4 \pm 1.6 \\
(1.4-8.9)\end{array}$ & $\begin{array}{l}1.7 \pm 0.78 \\
(0.30-3.3)\end{array}$ & $\begin{array}{l}28 \pm 2.0 \\
(24-33)\end{array}$ & $\begin{array}{c}0.057 \pm 0.0096 \\
(0.037-0.079)\end{array}$ & $\begin{array}{c}0.23 \pm 0.076 \\
(0.14-0.45)\end{array}$ & $\begin{array}{c}5.9 \pm 0.81 \\
(3.9-7.3)\end{array}$ & $\begin{array}{c}83 \pm 9.4 \\
(62-100)\end{array}$ \\
\hline
\end{tabular}

was often abundant (mean and highest measured concentration in all samples: 11 and $37 \mu \mathrm{mol} \mathrm{g}{ }^{-1}$, respectively), although TauB was not extracted consistently from clam tissues. Betaine concentrations in the samples of clam zooxanthellae are presented in Table 4, normalized against pheophytin concentrations.

DMSP concentration was measured in 24 tridacnid tissue specimens. In adult T. maxima, DMSP concentration averaged 3.2 (range: $2.0-5.2$ ) $\mu_{m o l ~ g^{-1}}$ in adductor, 70 (46-91) $\mathrm{\mu mol} \mathrm{g}^{-1}$ in byssal mantle, 46 (40-56) $\mathrm{mmol} \mathrm{g}^{-1}$ in gill, and 34 (15-56) $\mu_{\mathrm{mol} \mathrm{g}}^{-1}$ in siphonal mantle ( $\mathrm{n}=3,4,4$, and 5 , respectively). In adult T. crocea, DMSP averaged 6.2 (2.6-9.8) $\mu \mathrm{mol}$ $\mathrm{g}^{-1}$ in adductor, 27 (25-29) $\mathrm{\mu mol} \mathrm{g}^{-1}$ in gill, and $19 \mu \mathrm{mol} \mathrm{g}^{-1}$ in siphonal mantle $(\mathrm{n}=2,2$, and 1$)$. DMSP concentrations in single specimens of adult $H$. hippopus siphonal mantle, juvenile T. maxima sipho- nal mantle, and juvenile T. crocea adductor were 10, 73, and $13 \mu \mathrm{mol} \mathrm{g}{ }^{-1}$, respectively.

\section{Betaines in wild coral tissues}

Measured amounts of betaines and other compounds in corals were normalized to coral surface area, and we refer to the normalized values as 'concentrations.' To assess whether the foil and waxdipping methods of coral area measurement yielded similar results, we measured the areas of 15 specimens of Montipora digitata by both methods. No significant difference between methods was observed (paired $t$-test, df $=14, \mathrm{p}=0.69$ ).

The concentrations of all measured betaines and pheophytin in wild-collected corals are summarized 
Table 3. Mean \pm SE (range) tissue concentrations of the 6 most abundant betaines and pheophytin in tissues of juvenile tridacnid clams of 3 species. HProB: hydroxyproline betaine. See Table 1 for definitions of other chemical abbreviations. n: number of samples analyzed, each sample taken from a distinct individual. Some tissues are not represented or have low sample size either because the tissue was too small to sample in some individuals, or sampling was limited by limits on cryogenic transport

\begin{tabular}{|c|c|c|c|c|c|c|c|}
\hline Tissue & $\begin{array}{c}\text { AValB } \\
\left(\mu \mathrm{mol} \mathrm{g}^{-1}\right)\end{array}$ & $\begin{array}{c}\beta A l a B \\
\left.(\mu \mathrm{mol} \mathrm{g})^{-1}\right)\end{array}$ & $\begin{array}{c}\text { GlyB } \\
\left.(\mu \mathrm{mol} \mathrm{g})^{-1}\right)\end{array}$ & $\begin{array}{c}\text { HProB } \\
\left(\mu \mathrm{mol} \mathrm{g}{ }^{-1}\right)\end{array}$ & $\begin{array}{c}\text { ProB } \\
\left.(\mu \mathrm{mol} \mathrm{g})^{-1}\right)\end{array}$ & $\begin{array}{l}\text { Trig/Hom } \\
\left(\mu \mathrm{mol} \mathrm{g}{ }^{-1}\right)\end{array}$ & $\begin{array}{c}\text { Pheophytin } \\
\left(\mu \mathrm{g} \mathrm{g}^{-1}\right)\end{array}$ \\
\hline \multicolumn{8}{|l|}{ Tridacna maxima } \\
\hline Adductor $(\mathrm{n}=6)$ & $\begin{array}{l}38 \pm 2.8 \\
(27-45)\end{array}$ & $\begin{array}{c}0.51 \pm 0.024 \\
(0.44-0.60)\end{array}$ & $\begin{array}{l}46 \pm 3.7 \\
(37-62)\end{array}$ & $\begin{array}{l}1.1 \pm 0.29 \\
(0.28-2.4)\end{array}$ & $\begin{array}{c}2.9 \pm 0.44 \\
(1.1-4.4)\end{array}$ & $\begin{array}{l}33 \pm 2.6 \\
(24-40)\end{array}$ & $\begin{array}{l}3.2 \pm 0.91 \\
(0.76-6.9)\end{array}$ \\
\hline Gill $(n=1)$ & 0.80 & 4.1 & 41 & 0.10 & 0.49 & 12 & 12 \\
\hline $\begin{array}{l}\text { Siphonal mantle } \\
(\mathrm{n}=8)\end{array}$ & $\begin{array}{l}14 \pm 1.9 \\
(4.7-23)\end{array}$ & $\begin{array}{l}8.3 \pm 1.1 \\
(5.6-15)\end{array}$ & $\begin{array}{l}12 \pm 1.9 \\
(6.4-24)\end{array}$ & $\begin{array}{l}1.0 \pm 0.25 \\
(0.20-2.3)\end{array}$ & $\begin{array}{c}0.64 \pm 0.093 \\
(0.26-0.99)\end{array}$ & $\begin{array}{l}13 \pm 2.1 \\
(8.3-26)\end{array}$ & $\begin{array}{c}285 \pm 44 \\
(167-561)\end{array}$ \\
\hline \multicolumn{8}{|l|}{ T. crocea } \\
\hline Adductor $(\mathrm{n}=6)$ & $\begin{array}{l}33 \pm 7.5 \\
(7.4-62)\end{array}$ & $\begin{array}{c}0.37 \pm 0.030 \\
(0.24-0.44)\end{array}$ & $\begin{array}{l}70 \pm 4.6 \\
(57-87)\end{array}$ & $\begin{array}{l}4.8 \pm 1.6 \\
(0.93-12)\end{array}$ & $\begin{array}{l}1.3 \pm 0.13 \\
(0.89-1.7)\end{array}$ & $\begin{array}{l}37 \pm 4.9 \\
(23-51)\end{array}$ & $\begin{array}{c}2.6 \pm 0.52 \\
(1.5-4.3)\end{array}$ \\
\hline $\begin{array}{l}\text { Byssal mantle } \\
(\mathrm{n}=3)\end{array}$ & $\begin{array}{l}14 \pm 4.3 \\
(7.8-22)\end{array}$ & $\begin{array}{c}4.1 \pm 0.27 \\
(3.6-4.5)\end{array}$ & $\begin{array}{l}24 \pm 4.7 \\
(19-33)\end{array}$ & $\begin{array}{l}1.7 \pm 0.47 \\
(0.96-2.6)\end{array}$ & $\begin{array}{c}0.46 \pm 0.098 \\
(0.29-0.63)\end{array}$ & $\begin{array}{l}9.8 \pm 2.7 \\
(6.8-15)\end{array}$ & $\begin{array}{l}13 \pm 1.4 \\
(10-15)\end{array}$ \\
\hline $\begin{array}{l}\text { Siphonal mantle } \\
(\mathrm{n}=6)\end{array}$ & $\begin{array}{l}25 \pm 5.3 \\
(13-44)\end{array}$ & $\begin{array}{l}7.9 \pm 0.46 \\
(6.6-9.8)\end{array}$ & $\begin{array}{l}19 \pm 1.7 \\
(12-25)\end{array}$ & $\begin{array}{l}1.6 \pm 0.32 \\
(0.67-2.6)\end{array}$ & $\begin{array}{c}0.61 \pm 0.050 \\
(0.49-0.81)\end{array}$ & $\begin{array}{l}9.7 \pm 1.1 \\
(6.2-13)\end{array}$ & $\begin{array}{c}261 \pm 36 \\
(129-351)\end{array}$ \\
\hline \multicolumn{8}{|l|}{ T. derasa } \\
\hline Adductor (n = 8) & $\begin{array}{l}44 \pm 6.6 \\
(32-88)\end{array}$ & $\begin{array}{l}1.4 \pm 0.16 \\
(0.77-2.0)\end{array}$ & $\begin{array}{l}45 \pm 6.0 \\
(15-64)\end{array}$ & $\begin{array}{c}0.60 \pm 0.044 \\
(0.51-0.90)\end{array}$ & $\begin{array}{c}3.8 \pm 0.73 \\
(1.7-8.3)\end{array}$ & $\begin{array}{l}26 \pm 3.7 \\
(16-47)\end{array}$ & $\begin{array}{c}3.3 \pm 0.44 \\
(1.3-4.6)\end{array}$ \\
\hline Gill $(\mathrm{n}=1)$ & 0.87 & 2.4 & 83 & 0.063 & 0.73 & 28 & 3.9 \\
\hline $\begin{array}{l}\text { Siphonal mantle } \\
(\mathrm{n}=9)\end{array}$ & $\begin{array}{l}14 \pm 1.7 \\
(6.4-22)\end{array}$ & $\begin{array}{c}5.0 \pm 0.41 \\
(3.9-7.2)\end{array}$ & $\begin{array}{l}14 \pm 1.4 \\
(9.3-23)\end{array}$ & $\begin{array}{l}0.18 \pm 0.041 \\
(0.064-0.48)\end{array}$ & $\begin{array}{c}0.33 \pm 0.031 \\
(0.16-0.42)\end{array}$ & $\begin{array}{l}16 \pm 1.3 \\
(9.9-23)\end{array}$ & $\begin{array}{c}257 \pm 38 \\
(118-480)\end{array}$ \\
\hline
\end{tabular}

in Table 5. TauB is included because it is extracted efficiently and consistently in corals (Hill et al. 2010).

\section{Betaines and skeletal density during coral acclimatization}

Betaine concentrations in the fragments of maricultured A. cerealis and M. digitata preserved in Pohnpei are presented in Table 6 . The living fragments of each species shipped to MSU (i.e. those that founded the acclimatization study) were held in our sea table for $14 \mathrm{~d}$ after arrival before first sampling. At that

Table 4. Mean \pm SE (range) concentrations normalized to pheophytin of betaines in zooxanthellae of adult tridacnid clams of 3 species. HProB: hydroxyproline betaine. See Table 1 for definitions of other chemical abbreviations

\begin{tabular}{|lccc|}
\hline & \multicolumn{3}{c|}{ Concentration $\left(\mathrm{nmol} \mathrm{ug}^{-1}\right.$ pheophytin) } \\
\cline { 2 - 4 } Compound & $\begin{array}{c}\text { Tridacna maxima } \\
(\mathrm{n}=9)\end{array}$ & $\begin{array}{c}\text { T. squamosa } \\
(\mathrm{n}=1)\end{array}$ & $\begin{array}{c}\text { Hippopus hippopus } \\
(\mathrm{n}=2)\end{array}$ \\
& $2.2 \pm 0.48(1.1-5.2)$ & 0.37 & $2.7(2.4-3.0)$ \\
AValB & $2.1 \pm 0.43(1.0-5.0)$ & 1.3 & $2.1(0.67-3.6)$ \\
BAlaB & $4.5 \pm 0.66(1.9-7.6)$ & 2.0 & $4.9(4.3-5.4)$ \\
GlyB & $0.050 \pm 0.011(0.017-0.12)$ & 0.018 & $0.074(0.041-0.11)$ \\
HProB & $0.16 \pm 0.045(0.080-0.52)$ & 0.058 & $0.55(0.41-0.70)$ \\
ProB & $1.7 \pm 0.27(0.74-3.1)$ & 0.50 & $2.1(1.6-2.5)$ \\
Trig/Hom & & & \\
\hline
\end{tabular}

time, there were no differences ( $t$-tests; $p>0.17$ for A. cerealis, $\mathrm{p}>0.05$ for $M$. digitata) between the fragments shipped alive and those preserved in Pohnpei (Table 6).

The fragments of $M$. digitata grew well during the acclimatization study, and following the first sampling at $14 \mathrm{~d}$ after establishment in the sea table, we took samples (target: $\mathrm{n}=6$ ) at 33, 67, 97, 132, 320, and $397 \mathrm{~d}$ after establishment. The A. cerealis fragments, however, were more variable in growth, and we took samples $(n=5-6)$ only at $14,33,320$, and 397 d. Bulk skeletal density could not be measured on some A. cerealis samples because of growth forms that precluded underwater weighing without bubbles.

Over the course of coral acclimatization, none of the measured variables increased or decreased in a steady manner that could be described with linear regression, as we had envisioned a priori. Thus, we needed to approach the acclimatization data with our most simple a priori hypothesis, namely that values at the end of the acclimatization period (397 d) were not different from those at the start (14 d). In A. cerealis, AlaB, AValB, 
Table 5. Mean \pm SE (range) concentrations of betaines and pheophytin in 6 scleractinian coral species collected in the wild at Pohnpei. n: number of independently collected specimens. HProB: hydroxyproline betaine, TauB: taurine betaine. See Table 1 for definitions of other chemical abbreviations. Porites massive: Porites lobata or Porites lutea, which could not be distinguished under our collecting conditions. All betaines are in units of $100 \times \mu \mathrm{mol} \mathrm{cm}{ }^{-2}$; divide listed value by 100 to obtain concentration in $\mu \mathrm{mol} \mathrm{cm}{ }^{-2}$. For pheophytin, divide listed value by 100 to obtain concentration in $\mathrm{mg} \mathrm{cm}^{-2}$

\begin{tabular}{|c|c|c|c|c|c|c|}
\hline Compound & $\begin{array}{c}\text { Acropora } \\
\text { formosa } \\
(\mathrm{n}=6)\end{array}$ & $\begin{array}{c}\text { Montipora } \\
\text { monasteriata } \\
\quad(\mathrm{n}=6)\end{array}$ & $\begin{array}{c}\text { Pavona } \\
\text { varians } \\
(\mathrm{n}=6)\end{array}$ & $\begin{array}{l}\text { Pocillopora } \\
\text { damicornis } \\
\quad(\mathrm{n}=7)\end{array}$ & $\begin{array}{l}\text { Porites } \\
\text { cylindrica } \\
(\mathrm{n}=8)\end{array}$ & $\begin{array}{c}\text { Porites } \\
\text { massive } \\
(\mathrm{n}=6)\end{array}$ \\
\hline AlaB & $\begin{array}{l}0.15 \pm 0.025 \\
(0.056-0.25)\end{array}$ & $\begin{array}{l}1.2 \pm 0.10 \\
(0.82-1.4)\end{array}$ & $\begin{array}{c}0.64 \pm 0.095 \\
(0.27-0.98)\end{array}$ & $\begin{array}{c}0.20 \pm 0.025 \\
(0.11-0.28)\end{array}$ & $\begin{array}{l}11 \pm 1.3 \\
(7.0-16)\end{array}$ & $\begin{array}{l}19 \pm 1.9 \\
(13-25)\end{array}$ \\
\hline AValB & $\begin{array}{c}0.31 \pm 0.10 \\
(0.11-0.70)^{\mathrm{a}}\end{array}$ & $\begin{array}{l}1.0 \pm 0.21 \\
(0.35-1.6)\end{array}$ & $\begin{array}{l}1.2 \pm 0.27 \\
(0.55-2.3)\end{array}$ & $\begin{array}{l}1.6 \pm 0.90 \\
(0.20-6.8)\end{array}$ & $\begin{array}{c}2.8 \pm 0.65 \\
(0.76-5.8)^{b}\end{array}$ & $\begin{array}{l}2.3 \pm 0.59 \\
(0.40-3.8)\end{array}$ \\
\hline$\beta$ AlaB & $\begin{array}{c}1.7 \pm 0.18 \\
(1.2-2.4)\end{array}$ & $\begin{array}{c}2.3 \pm 0.25 \\
(1.4-3.2)\end{array}$ & $\begin{array}{l}1.5 \pm 0.47 \\
(0.42-3.4)\end{array}$ & $\begin{array}{c}0.62 \pm 0.11 \\
(0.24-1.0)\end{array}$ & $\begin{array}{l}2.9 \pm 0.30 \\
(2.1-3.8)^{b}\end{array}$ & $\begin{array}{l}4.1 \pm 0.98 \\
(1.7-6.0)^{\mathrm{c}}\end{array}$ \\
\hline GABAB & $\begin{array}{c}0.54 \pm 0.25 \\
(0.10-1.7)\end{array}$ & $\begin{array}{l}0.43 \pm 0.14 \\
(0.050-1.1)\end{array}$ & $\begin{array}{c}0.49 \pm 0.086 \\
(0.13-0.71)\end{array}$ & $\begin{array}{l}0.12 \pm 0.032 \\
(0.012-0.21)\end{array}$ & $\begin{array}{c}5.8 \pm 0.95 \\
(2.9-11)\end{array}$ & $\begin{array}{l}2.9 \pm 0.70 \\
(0.85-5.5)\end{array}$ \\
\hline GlyB & $\begin{array}{l}8.6 \pm 1.0 \\
(5.2-12)\end{array}$ & $\begin{array}{c}490 \pm 76 \\
(290-760)\end{array}$ & $\begin{array}{c}230 \pm 29 \\
(180-380)\end{array}$ & $\begin{array}{l}140 \pm 19 \\
(71-230)\end{array}$ & $\begin{array}{l}19 \pm 1.9 \\
(9.9-29)\end{array}$ & $\begin{array}{l}18 \pm 3.1 \\
(13-33)\end{array}$ \\
\hline HProB & $\begin{array}{c}0.41 \pm 0.077 \\
(0.18-0.67)\end{array}$ & $\begin{array}{l}17 \pm 4.5 \\
(1.1-34)\end{array}$ & $\begin{array}{l}4.4 \pm 2.3 \\
(0.35-15)\end{array}$ & $\begin{array}{l}1.3 \pm 0.40 \\
(0.18-2.9)\end{array}$ & $\begin{array}{l}23 \pm 1.8 \\
(16-30)\end{array}$ & $\begin{array}{l}20 \pm 5.1 \\
(4.9-38)\end{array}$ \\
\hline ProB & $\begin{array}{c}0.86 \pm 0.20 \\
(0.30-1.4)\end{array}$ & $\begin{array}{l}4.7 \pm 0.88 \\
(2.7-8.5)\end{array}$ & $\begin{array}{l}7.1 \pm 2.0 \\
(1.9-14)\end{array}$ & $\begin{array}{l}1.2 \pm 0.20 \\
(0.55-1.9)\end{array}$ & $\begin{array}{l}150 \pm 14 \\
(97-210)\end{array}$ & $\begin{array}{l}58 \pm 17 \\
(20-130)\end{array}$ \\
\hline TauB & $\begin{array}{l}0.67 \pm 0.24 \\
(0.077-1.7)\end{array}$ & $\begin{array}{c}9.4 \pm 4.4 \\
(0.68-29)\end{array}$ & $\begin{array}{c}3.0 \pm 1.0 \\
(0.37-6.4)\end{array}$ & $\begin{array}{c}0.54 \pm 0.21 \\
(0.18-1.8)\end{array}$ & $\begin{array}{l}63 \pm 5.0 \\
(45-84)\end{array}$ & $\begin{array}{c}84 \pm 8.6 \\
(49-110)\end{array}$ \\
\hline Trig/Hom & $\begin{array}{l}2.2 \pm 0.30 \\
(0.86-3.0)\end{array}$ & $\begin{array}{l}28 \pm 4.2 \\
(16-41)\end{array}$ & $\begin{array}{c}3.6 \pm 0.65 \\
(1.4-6.4)\end{array}$ & $\begin{array}{l}1.2 \pm 0.23 \\
(0.55-2.3)\end{array}$ & $\begin{array}{l}14 \pm 2.3 \\
(7.0-24)\end{array}$ & $\begin{array}{l}84 \pm 7.9 \\
(66-120)\end{array}$ \\
\hline Total betaines & $\begin{array}{l}15 \pm 1.6 \\
(10-21)\end{array}$ & $\begin{array}{c}560 \pm 79 \\
(310-810)\end{array}$ & $\begin{array}{c}260 \pm 33 \\
(180-410)\end{array}$ & $\begin{array}{l}140 \pm 20 \\
(74-240)\end{array}$ & $\begin{array}{c}290 \pm 20 \\
(200-380)\end{array}$ & $\begin{array}{l}290 \pm 16 \\
(230-330)\end{array}$ \\
\hline $\begin{array}{l}\text { Pheophytin } \\
\left(100 \times \mathrm{mg} \mathrm{cm}^{-2}\right)\end{array}$ & $\begin{array}{c}0.33 \pm 0.054 \\
(0.21-0.52)\end{array}$ & $\begin{array}{c}0.58 \pm 0.091 \\
(0.34-0.85)\end{array}$ & $\begin{array}{c}0.57 \pm 0.090 \\
(0.31-0.96)\end{array}$ & $\begin{array}{c}0.22 \pm 0.041 \\
(0.13-0.44)\end{array}$ & $\begin{array}{c}0.67 \pm 0.068 \\
(0.42-0.94)\end{array}$ & $\begin{array}{c}0.39 \pm 0.083 \\
(0.16-0.74)\end{array}$ \\
\hline${ }^{\mathrm{a}} \mathrm{n}=5 ;{ }^{\mathrm{b}} \mathrm{n}=7 ;{ }^{\mathrm{c}} \mathrm{n}$ & & & & & & \\
\hline
\end{tabular}

DMSP, ProB, and TauB underwent no change in mean concentration between Days 14 and 397 (t-tests). In M. digitata, AValB, GlyB, DMSP, GABAB, pheophytin, TauB, and Trig/Hom also underwent no change. In all these cases, visual inspection indicated that mean concentrations were steady at all sampling times.

However, at the end of the acclimatization study (Day 397), A. cerealis had higher concentrations of GlyB, $\beta A l a B, G A B A B$, and pheophytin than at the start (Day 14) (2-tailed $t$-tests planned a priori; $\mathrm{p}<$ 0.001 , except $\mathrm{p}<0.02$ for GABAB). Additionally, $A$. cerealis had lower concentrations at the end for HProB ( $1<0.05)$ and Trig/Hom $(p<0.01)$ (Table 7). Based on an a posteriori descriptive analysis, the 4 compounds that increased did so in parallel (Fig. 1). The concentrations of all 4 were far higher on Day 320 than Days 14-33; GlyB and pheophytin were 2.9-3.1 times higher, whereas $\beta \mathrm{AlaB}$ and GABAB were 1.9-2.3 times higher. The concentrations of all 4 compounds fell between Days 320 and 397 (Fig. 1) while remaining significantly higher than at the start. Of the 2 betaines that fell between start and end (Table 7), Trig/Hom appeared to fall progressively with the passage of time, whereas HProB changed in a less regular manner.

As Table 7 shows, $M$. digitata had lower concentrations of AlaB, $\beta$ AlaB, ProB, and HProB at the end of the acclimatization study than at the start (2-tailed $t$-tests; $\mathrm{p}<0.05$, except $\mathrm{p}<0.0001$ for HProB). Descriptively, HProB fell progressively over the entire study period, $\beta$ AlaB underwent most of its decline between the start and Day 132, and AlaB and ProB showed no regular temporal trends.

We had a directional a priori hypothesis for changes in bulk skeletal density during acclimatization and thus analyzed these results with 1-tailed statistical tests. Specifically, we hypothesized that bulk density would decrease during acclimatization, as Carlson (1999) reported. Our initial $t$-tests to compare start and end values indicated that bulk density in both species was lower on Day 397 than Day 14 ( $\mathrm{p}<$ 0.01). Bulk density is shown as a function of time in Fig. 2. Within each species, we analyzed the data for all sampling dates with 1-way ANOVA. In A. cerealis, the ANOVA indicated that days in captivity had a significant effect $(p<0.01)$, and a post hoc Student- 
Table 6. Mean \pm SE (range) concentrations of betaines and pheophytin - and bulk density of skeleton - in maricultured fragments of 2 scleractinian coral species that had been maintained in the Northeast Pohnpei Lagoon and preserved immediately after collection. n: number of fragments analyzed. HProB: hydroxproline betaine, TauB: taurine betaine. See Table 1 for definitions of other chemical abbreviations. All betaines are in units of $100 \times \mu \mathrm{mol} \mathrm{cm} \mathrm{c}^{-2}$; divide listed value by 100 to

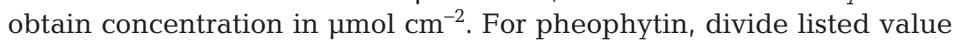
by 100 to obtain concentration in $\mathrm{mg} \mathrm{cm}^{-2}$

\begin{tabular}{|c|c|c|}
\hline $\begin{array}{l}\text { Compound or } \\
\text { other property }\end{array}$ & $\begin{array}{c}\text { Acropora cerealis } \\
\qquad(\mathrm{n}=6)\end{array}$ & $\begin{array}{c}\text { Montipora digitata } \\
(\mathrm{n}=6)\end{array}$ \\
\hline AlaB & $\begin{array}{c}0.12 \pm 0.0068 \\
(0.098-0.14)\end{array}$ & $\begin{array}{c}0.22 \pm 0.022 \\
(0.15-0.30)\end{array}$ \\
\hline AValB & $\begin{array}{c}13 \pm 5.7 \\
(0.30-32)\end{array}$ & $\begin{array}{l}10 \pm 4.8 \\
(1.7-29)\end{array}$ \\
\hline BAlaB & $\begin{array}{c}0.99 \pm 0.058 \\
(0.85-1.3)\end{array}$ & $\begin{array}{c}1.5 \pm 0.13 \\
(1.3-2.2)\end{array}$ \\
\hline GABAB & $\begin{array}{c}0.41 \pm 0.030 \\
(0.28-0.50)\end{array}$ & $\begin{array}{l}0.051 \pm 0.0086 \\
(0.024-0.072)^{b}\end{array}$ \\
\hline GlyB & $\begin{array}{c}6.3 \pm 0.36 \\
(5.4-7.8)\end{array}$ & $\begin{array}{c}240 \pm 16 \\
(180-290)\end{array}$ \\
\hline HProB & $\begin{array}{c}0.32 \pm 0.031 \\
(0.22-0.42)\end{array}$ & $\begin{array}{c}0.77 \pm 0.059 \\
(0.57-1.0)\end{array}$ \\
\hline ProB & $\begin{array}{c}0.35 \pm 0.033 \\
(0.26-0.45)\end{array}$ & $\begin{array}{l}1.6 \pm 0.18 \\
(1.0-2.0)\end{array}$ \\
\hline TauB & $\begin{array}{l}0.076 \pm 0.0062 \\
(0.066-0.088)^{\mathrm{a}}\end{array}$ & $\begin{array}{l}1.4 \pm 0.19 \\
(0.64-1.9)\end{array}$ \\
\hline Trig/Hom & $\begin{array}{c}1.8 \pm 0.11 \\
(1.6-2.2)\end{array}$ & $\begin{array}{l}5.5 \pm 1.1 \\
(2.3-9.7)\end{array}$ \\
\hline Pheophytin $\left(100 \times \mathrm{mg} \mathrm{cm}^{-2}\right)$ & $\begin{array}{c}0.26 \pm 0.016 \\
(0.22-0.33)\end{array}$ & $\begin{array}{c}0.76 \pm 0.071 \\
(0.52-0.95)\end{array}$ \\
\hline Bulk density $\left(\mathrm{g} \mathrm{ml}^{-1}\right.$ ) & $\begin{array}{c}1.1 \pm 0.027 \\
(1.0-1.2)\end{array}$ & $\begin{array}{c}0.78 \pm 0.070 \\
(0.57-1.0)\end{array}$ \\
\hline${ }^{\mathrm{a}} \mathrm{n}=3 ;{ }^{\mathrm{b}} \mathrm{n}=5$ & & \\
\hline
\end{tabular}

M. digitata, days in captivity had a significant effect ( $p<0.01)$, and post hoc analysis indicated that the densities on Days 320 and 397 were homogeneous and significantly lower than those on Days 14, 33, 97, and 137 .

\section{DISCUSSION}

Betaines are abundant metabolites in tridacnid clams, although their presence in tridacnids was heretofore unknown. Tissue millimolar concentrations in tridacnids can be estimated as being numerically equal to the values we report here in $\mu$ mol $\mathrm{g}^{-1}$ (Tables 2 \& 3). By this standard, total betaine concentrations in tridacnid tissues are usually $>50 \mathrm{mM}$ and sometimes reach 150-250 mM. In corals, tissue millimolar concentrations can be estimated from areanormalized concentrations by assuming a model tissue thickness of $1 \mathrm{~mm}$ (Hill et al. 2010). On this basis, the tissue millimolar concentration of a betaine in a coral is numerically equal to $10 \%$ of the value reported in Tables 5-7 in units of $100 \times$ $\mu \mathrm{mol} \mathrm{cm} \mathrm{cm}^{-2}$ (Hill et al. 2010). Thus we estimate total betaine concentrations in our wild-collected corals to be 14-56 (mean = 31) $\mathrm{mM}$ in all but Acropora formosa (Table 5). Studies of other photosynthetic organisms

Table 7. Statistically significant decreases in tissue concentrations (mean \pm SE) between start (Day 14) and end (Day 397) of coral acclimatization study. For Acropora cerealis, start: $\mathrm{n}=5$, end: $\mathrm{n}=6$. For Montipora digitata, start: $\mathrm{n}=$ 5, end: $\mathrm{n}=7$. HProB: hydroxyproline betaine. See Table 1 for definitions of other chemical abbreviations. Units are $100 \times$

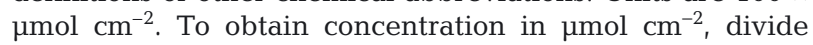
listed value by 100

\begin{tabular}{|c|c|c|c|}
\hline Species & $\begin{array}{l}\text { Com- } \\
\text { pound }\end{array}$ & $\begin{array}{l}\text { Concer } \\
(100 \times \mu \mathrm{mol} \mathrm{cm} \\
\text { Start (Day 14) }\end{array}$ & $\begin{array}{l}\text { tration } \\
-2 \text { ), mean } \pm \mathrm{SE} \\
\text { End (Day 397) }\end{array}$ \\
\hline A. cerealis & $\begin{array}{l}\text { HProB } \\
\text { Trig/Hom }\end{array}$ & $\begin{aligned} 0.32 & \pm 0.029 \\
1.6 & \pm 0.24\end{aligned}$ & $\begin{array}{l}0.24 \pm 0.018 \\
0.86 \pm 0.048\end{array}$ \\
\hline M. digitata & $\begin{array}{l}\text { AlaB } \\
\text { BAlaB } \\
\text { ProB } \\
\text { HProB }\end{array}$ & $\begin{array}{c}0.21 \pm 0.021 \\
1.3 \pm 0.13 \\
1.1 \pm 0.17 \\
0.66 \pm 0.034\end{array}$ & $\begin{array}{l}0.14 \pm 0.016 \\
0.88 \pm 0.086 \\
0.67 \pm 0.10 \\
0.30 \pm 0.025\end{array}$ \\
\hline
\end{tabular}

Newman-Keuls analysis indicated that the densities on Days 320 and 397 were homogeneous and significantly lower than the densities on Days 14 and 33. In indicate that the tissue concentrations thus estimated in tridacnids and western Pacific corals are of sufficient magnitude for stabilization of protein and membrane functions (Prasad \& Saradhi 2004, Shirasawa et al. 2006, Yang et al. 2007, Chen \& Murata 2011). In actuality, the true, operative millimolar concentrations are likely to be substantially higher than those estimated here, because our methods of estimation assume a homogeneous subcellular distribution, whereas betaines are probably, in fact, more concentrated in some subcellular regions than others.

According to the extensive literature on betaine actions in terrestrial plants and free-living algae referenced in the 'Introduction' and elsewhere in this paper, betaines exert a number of beneficial effects on cellular function by stabilizing proteins and membranes (Rhodes \& Hanson 1993, McNeil et al. 1999). These protein- and membrane-stabilizing effects are attributed for the most part to influences that betaines exert on the structure of water in the immediate vicinity of protein molecules (influences medi- 


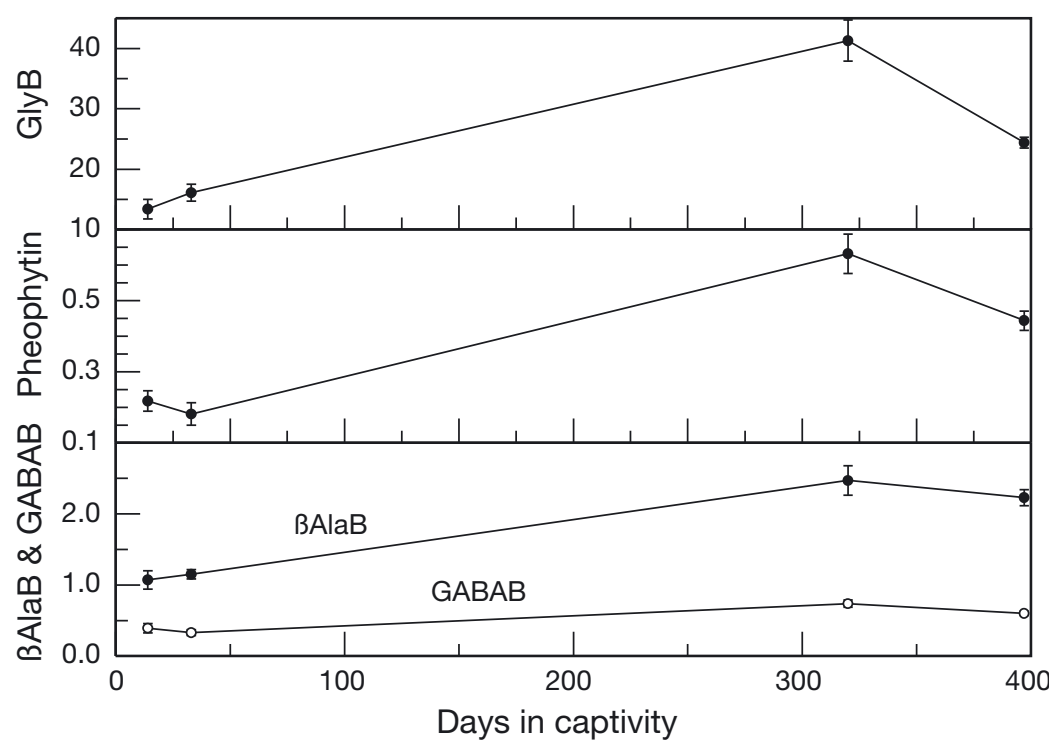

Fig. 1. Statistically significant increases in tissue concentrations of 3 betaines $\left(100 \times \mu \mathrm{mol} \mathrm{cm}{ }^{-2}\right.$; mean $\left.\pm \mathrm{SE}\right)$ and pheophytin $\left(100 \times \mathrm{mg} \mathrm{cm}^{-2}\right.$; mean $\left.\pm \mathrm{SE}\right)$ as functions of days in captivity in Acropora cerealis during coral acclimatization study. To obtain concentrations in $\mu \mathrm{mol} \mathrm{cm}$ (betaines) or $\mathrm{mg} \mathrm{cm}^{-2}$ (pheophytin), divide plotted values by 100 . Lines are for practical purposes only and not intended to imply patterns of change between observed data points. $\mathrm{n}=5$ on Days 14 and 33, $\mathrm{n}=6$ on Days 320 and 397. $\beta$ AlaB: $\beta$-alanine betaine,

GABAB: $\gamma$-aminobutyric acid betaine, GlyB: glycine betaine

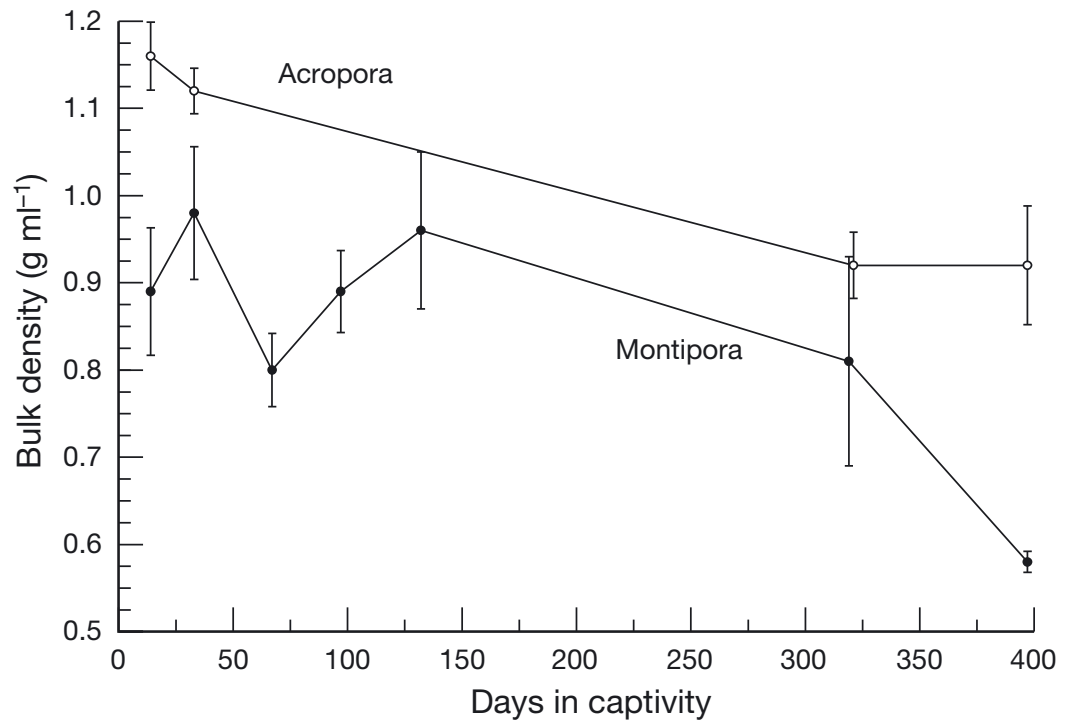

Fig. 2. Bulk density of skeleton (mean $\pm \mathrm{SE}$ ) as function of days in captivity in coral acclimatization study. Lines are for practical purposes only and not intended to imply patterns of change between observed data points. For Acropora cerealis, $\mathrm{n}=5$ on Days 14 and 397, $\mathrm{n}=4$ on Day 33, and $\mathrm{n}=3$ on Day 320. For Montipora digitata, $\mathrm{n}=6$ on all days, except $\mathrm{n}=5$ on Day 14 and $\mathrm{n}=7$ on Day 397

ated by noncovalent betaine-protein and betainewater interactions) (McNeil et al. 1999, Bennion \& Daggett 2004, Street et al. 2006, Auton et al. 2011, Guinn et al. 2011, Roychoudhury et al. 2013). Specif- ically, in the presence of betaines, water structure is altered in ways that enhance the extent to which native protein molecular states are more favorable thermodynamically than nonnative states. Existing theories of betaine action, such as these, stress universal betaine properties. Thus, different chemical species of betaines are often viewed as being relatively interchangeable and additive in their effects (Rhodes \& Hanson 1993, Yancey 2005). On the other hand, Anthoni et al. (1991) predicted that biologists ultimately will recognize that natural selection has differentially favored various betaines in various contexts because of distinctive, compound-specific properties. Understanding of compound-specific properties, however, remains in its early infancy.

One of the most significant results reported here is the discovery that betaines occur in abundance in the tissues of all 5 species of tridacnid clams studied. We originally hypothesized that betaines would be present in tridacnids because of the clams' similarities to reef-building corals: The clams resemble corals in being symbiotic with Symbiodinium zooxanthellae, and the clams live in the same communities as corals-under closely similar environmental conditions. We have found, in fact, that some tridacnid tissues (Tables $2 \& 3$ ) have total betaine concentrations (estimated millimolarities) that rank with the highest values yet observed in reef-building corals $\left(\sim 200 \mathrm{mmol} \mathrm{l}^{-1}\right.$; Hill et al. 2010). However, we had originally hypothesized that among tridacnid tissues, the upward-facing, siphonal mantle would be highest in betaine concentrations because the zooxanthellae are located there, but that hypothesis is not supported by the data (Tables $2 \& 3$ ). We studied 2 tridacnid species (Tridacna maxima and T. crocea) as both adults and juveniles. In each of these species, relative concentrations of the major betaines - AValB, GlyB, and Trig/Hom - are generally similar, both within and among tissue types, in 
the adults (Table 2) and juveniles (Table 3). T. derasa adults seem likely therefore to have relative betaine profiles similar to those in the $T$. derasa juveniles studied (Table 3).

Adult tridacnid concentrations of DMSP are in general closely similar to those previously reported (R. W. Hill et al. 2004). The DMSP concentrations measured in juvenile tridacnids, although sparse $(n=2)$, are 2.5-4 times higher than average concentrations in the same tissues of adults (R. W. Hill et al. 2004), suggesting that study of ontogenetic changes in tridacnid DMSP may be revealing.

Comparing tridacnids with corals, bold differences exist in the dominant chemical species of betaines. Our study of betaines in Caribbean corals (Hill et al. 2010) was the first to measure the concentrations of multiple chemical species of betaines in reef animals using compound-specific standards (Li et al. 2010). In that study, which encompassed 10 species (6 genera) of corals, AValB was universally so low in concentration that we did not report it. The AValB concentration is also very low in most of the Pacific corals studied here (Table 5). It is thus striking that AValB is often a major betaine in tridacnid tissues (Tables $2 \& 3$ ). Contrariwise, whereas ProB is often a major betaine in corals (Hill et al. 2010; our Table 5), it is present at relatively low concentration in all 5 tridacnid species (Tables 2 \& 3). These sharp contrasts between tridacnids and corals in the chemical species of betaines suggest that natural selection has differentially favored various betaines in various contexts, as Anthoni et al. (1991) predicted. An alternative hypothesis would be that tridacnids and corals differ biochemically in ways that canalize betaine synthesis differentially.

Regarding betaines in the zooxanthellae of tridacnids, we realized a priori that the measurements we could make under the constrained options for tissue processing in Pohnpei would be largely limited to betaine presence or absence. The key insight provided by the data (Table 4 ) is that multiple betaines are present in association with the zooxanthellae. We stress that our zooxanthellae betaine concentrations normalized to pheophytin cannot be meaningfully compared with betaine-to-pheophytin ratios in the whole siphonal mantle tissue where the zooxanthellae live (Tables $2 \& 3$ ). This is true because all tissue cells in the mantle probably contain betaines, even though only the zooxanthellae contain pheophytin, making for high ratios of betaines to pheophytin in whole siphonal tissue. Notably, betaine concentrations in the zooxanthellae normalized to pheophytin tend to be in the same ranges across all 3 species of tridacnids studied (Table 4).
Turning our focus to corals, Fukami et al. (2004) have demonstrated that deep evolutionary divergence exists between Pacific and Atlantic reef corals. Thus, despite detailed knowledge of a wide range of betaines in Atlantic corals (Hill et al. 2010), it has been important to study Pacific corals in their own right using similar methods. The very first studies of betaines in corals were non-quantitative studies of GlyB in Pacific corals by Moore \& Huxley (1976) and Suenaga (2004). They reported that unspecified Pacific Acropora and Montipora contained GlyB and suggested that GlyB is a possible agent of attraction or repulsion for corallivores. Yancey et al. (2010) expanded knowledge of Pacific coral betaines by demonstrating that ProB is present in addition to GlyB and by quantifying those 2 compounds. Here we expand knowledge of Pacific corals further by quantifying not only GlyB and ProB, but also 7 additional betaines (Tables 5-7). As explained in Hill et al. (2010), we placed a top priority on processing tissue to the minimal extent required for high-quality metabolite quantification, so as to minimize opportunities for processing artifacts. Thus we studied the coral tissue in its entirety, rather than subjecting it to the sequence of extra procedures required to divide it into 2 or more subcellular constituents.

A significant difference between our results on Pacific corals and those of Yancey et al. (2010) concerns the question of whether GlyB and ProB are mutually exclusive. Yancey et al. (2010) found that the 2 compounds are mutually exclusive (no ProB in species that have GlyB, no GlyB in species having ProB). However, all the individual specimens of corals we studied (thus, all species) had both GlyB and ProB simultaneously present in readily detectable abundance. In Pocillopora damicornis, we find GlyB to be high in concentration and ProB low (Table 5), whereas Yancey et al. (2010) reported GlyB to be high but ProB to be undetectable in both Pocillopora species they studied. In both species of Porites we studied, we find ProB to be high and GlyB to be lower but still present at concentrations 13-31\% as high as ProB (Table 5). In contrast, Yancey et al. (2010) reported ProB to be high but GlyB to be absent in both Porites species they studied. In the 2 Montipora species we studied, we found notably high values for GlyB and values for ProB only $1 \%$ as high. Yancey et al. (2010) concur, except that they found ProB to be undetectable. To explain the differences in results, the most important factor seems likely to be that we used LC-MS for our measurements, a method noted for its high sensitivity and resolution. 
With LC-MS, we have detected 9 betaines in each species of Pacific coral studied, meaning Pacific corals resemble Atlantic corals (Hill et al. 2010) in having complex betaine profiles. In Pacific corals as in Atlantic, GlyB and ProB typically dominate, in the sense that one or the other, or both, are usually the most concentrated betaines (Table 5). However, as is also true in Atlantic corals, there are exceptional Pacific species that have other betaines in notable abundance. The genus Porites is especially notable. In the Atlantic data set, Porites astreoides stood out from 8 other (non-Porites) species in having (in addition to GlyB and ProB) dramatically high concentrations of certain betaines (AlaB, HProB, and TauB) that are usually present in corals at only low concentrations (Hill et al. 2010). In striking parallel, in the 2 Pacific Porites species studied here, AlaB, HProB, TauB, and Trig/Hom are abundant betaines, in contrast to their relatively low concentrations in most other Pacific species (Tables 5 \& 6).

Our data on Pacific corals point to taxon-specific divergences in betaine profiles in other ways as well. Focusing on divergences between genera, for example, both Pacific Acropora species studied (Tables 5 \& 6) are dramatically lower in total betaines than other Pacific corals. Also, as already implied, ratios of GlyB to ProB differ between genera: GlyB high relative to ProB in both Montipora species studied, ProB high relative to GlyB in both Porites studied (Tables 5 $\& 6)$. The low betaine concentrations in Acropora species may be a contributing factor to the observed sensitivity of acroporid corals to bleaching (Baird \& Marshall 2002).

One goal of our research was to clarify if there are biogeographical trends within taxa of corals. In Micronesia, we studied all 3 genera of zooxanthellate corals (Montipora, Pocillopora, and Porites) in which Yancey et al. (2010) measured GlyB and ProB in Hawaii, including 2 species that Yancey et al. (2010) also studied, Pocillopora damicornis and Porites lobata (our 'Porites massive'). In general, our results on GlyB and ProB in the western Pacific agree with the measurements on GlyB and ProB obtained by Yancey et al. (2010) in Hawaii, indicating that species maintain consistent betaine profiles across broad geographical ranges.

Pacific corals that are high in ProB concentration tend to have far lower ProB concentrations than ProB-rich Atlantic corals. The ProB concentrations we report here for Pacific Porites spp. in Micronesia, 6-15 mmol $\mathrm{l}^{-1}$ (Table 5), are closely similar to those Yancey et al. (2010) reported in Hawaii, 9-18 mmol $\mathrm{l}^{-1}$ (see Hill et al. 2010 for methods to compare the 2 sets of results). In contrast, the ProB-rich Atlantic corals studied by Hill et al. (2010), including Porites astreoides as well as 2 Madracis species, had ProB concentrations that were an order of magnitude higher, 100-180 mmol $\mathrm{l}^{-1}$.

We had 2 reasons for studying laboratory acclimatization of coral betaine concentrations. First, acclimatization in coral defense systems has been documented (Edmunds \& Gates 2008), including acclimatization of protection of photosynthesis and PSII (Brown et al. 2002, Middlebrook et al. 2008). Betaines may play central roles in photosystem defenses (see 'Introduction') and thus may be mediators of defense system acclimatization. Second, we wanted to learn whether cultured corals might be equivalent to wild corals for future studies of betaine functional roles. Because we knew little about betaines in Pacific corals when we started the acclimatization study, it was pure happenstance that, of the 2 species we chose for study, one-A. cerealis - is among the lowest in total betaines of all corals on which measurements have been made (Tables 5 \& 6).

A. cerealis exhibited dramatic upregulation of 3 betaines and chlorophyll during the year of laboratory acclimatization (Fig. 1). The concentrations of GlyB and chlorophyll, for example, increased 3-fold. We propose that the most parsimonious explanation of these changes is that (1) the density of zooxanthellae increased in the coral tissues during acclimatization, and (2) the 3 betaines shown in Fig. 1 are associated with the zooxanthellae, thereby increasing in the same or similar proportions as chlorophyll. In this way, the acclimatization results may support the hypothesis that betaines are associated with coral photosystems. All other significant changes in betaine concentrations during acclimatization, in both species studied, were decreases in the concentrations of betaines that are present at relatively low concentrations (Table 7).

Bulk skeletal density decreased significantly during acclimatization in both $A$. cerealis and Montipora digitata (Fig. 2). Carlson (1999) reported that aquarists at display aquariums sometimes observe reduced skeletal density and increased fragility in captive corals. He quantified bulk density in A. microphthalma and A. pulchra, and found that specimens in captivity for 6 yr were dramatically less dense than wild conspecifics. Our results suggest that reduced skeletal density may be a consistent feature of acclimatization to captive conditions.

What functional roles do betaines play in tridacnids and reef corals? By far the oldest hypothesis is that 
betaines are important in reef sensory ecology. The basis for this hypothesis is that some fish and invertebrates respond behaviorally to low ambient concentrations of GlyB (Kasumyan \& Døving 2003), and at least 1 corallivore (Acanthaster planci) exhibits a predation-behavior response to GlyB (Moore \& Huxley 1976). Yancey et al. (2010) argue for an important osmolyte function for coral betaines.

We urge that for a full understanding of betaine function in tridacnids and reef corals, the symbiotic nature of these animals will likely be an essential focus. Both groups are zooxanthellate, and they coexist in high-irradiance coral reef ecosystems where they are subject to critical photosystem stresses: photoinhibition and bleaching (Leggat et al. 2003, Franklin et al. 2006, Lesser 2011). With these facts in mind, the large literature on betaines as protective metabolites in terrestrial plants and free-living algae - reviewed in the 'Introduction' - seems likely to provide instructive guidance (Lesser 2011) for understanding betaine functions in tridacnids and corals.

In parallel with terrestrial plants, healthy reefbuilding corals commonly experience photoinhibition under high irradiance (Gorbunov et al. 2001, Jones \& Hoegh-Guldberg 2001, Winters et al. 2003, Franklin et al. 2006, Hoogenboom et al. 2006). PSII in the algal symbionts of the corals is the principal locus of this photoinhibition (Gorbunov et al. 2001, Jones \& Hoegh-Guldberg 2001), just as PSII is implicated in photoinhibition in plants. In fact, similarities in the mechanism of photoinhibition in corals and plants are increasingly apparent (Takahashi \& Murata 2008). Disruption of PSII in the algal symbionts of corals is also implicated in bleaching; during coral bleaching, the primary insult is often to PSII or to pathways of electron flow downstream from PSII in the algal symbionts (Warner et al. 1999, Fitt et al. 2001, Jones \& Hoegh-Guldberg 2001, R. Hill et al. 2004, Lesser \& Farrell 2004).

The concordance of concepts is striking: betaines are documented to protect PSII in many non-coral photosynthetic organisms, and much evidence indicates that PSII in the algal symbionts of corals is a primary target of photodamage during photoinhibition and bleaching. Where we refer to PSII, we intend to encompass the reaction center, the oxygen-evolving complex, the immediate milieu in the thylakoid membrane, and processes of post-stress repair thereof. Betaines can exert protective effects at multiple molecular sites within this suite (Allakhverdiev et al. 2008). Thus, betaines could be active under several different detailed scenarios of PSII function.
The potential importance of betaines in ameliorating photosystem stress in tridacnids and corals is further emphasized by additional observations on plants and free-living algae. Betaines have been demonstrated to reduce photoinhibition in crop plants (Prasad \& Saradhi 2004, Yang et al. 2007, Li et al. 2014, Wang et al. 2014). Betaines also have been demonstrated to increase photosystem defenses against reactive oxygen species (ROS) in both crop plants and free-living algae (Prasad \& Saradhi 2004, Hema et al. 2007, Yang et al. 2007, Chen \& Murata 2011, Fan et al. 2012). ROS are widely implicated in photodamage in corals (Lesser 2011), suggesting again that coral betaines play defensive roles.

A paramount question for future research is the metabolic source of tridacnid and coral betaines, i.e. whether they are synthesized by the animal tissue, the algal symbionts, or both the animal tissue and symbionts. GlyB is well documented to be synthesized by a wide variety of both algae and animals (Anthoni et al. 1991, Blunden et al. 1992, Keller et al. 1999, McNeil et al. 1999, Yancey 2005, Yancey et al. 2010), including mussels and oysters among bivalve molluscs (Perrino \& Pierce 2000, de Vooys \& Geenevasen 2002). These facts suggest that in tridacnids and corals - speaking in general-the metabolic source of GlyB could be animal, algal, or both. The same seems likely for at least some other betaines. Other paramount questions for future research include the subcellular distribution of betaines and the mechanisms that regulate betaine accumulation in animal and algal tissues.

Acknowledgements. We dedicate this paper to Ahser Edward, a magnificent colleague, who was born on Pingelap (Federated States of Micronesia) and later died there after a life of many contributions that ended in uncommon tragedy. A. D. Jones provided access to mass spectrometry. Lori Colin provided essential planning assistance and access to work space at the Coral Reef Research Foundation (Koror). Lihno Panuelo provided expert assistance at sea (Pohnpei). Essential support for the acclimatization study came from A\&M Aquatics (Lansing, MI), Simon Ellis, William Backus, Brian Wagner, and Ryan Shelander. Bong Dilanco, Marion Henry, Susan Hill, and Walt Peebles also provided invaluable help.

\section{LITERATURE CITED}

Alia, Hayashi H, Sakamoto A, Murata N (1998) Enhancement of the tolerance of Arabidopsis to high temperatures by genetic engineering of the synthesis of glycinebetaine. Plant J 16:155-161

Allakhverdiev SI, Hayashi H, Nishiyama Y, Ivanov AG and others (2003) Glycinebetaine protects the D1/D2/Cyt b559 complex of photosystem II against photo-induced and heat-induced inactivation. J Plant Physiol 160:41-49 
Allakhverdiev SI, Kreslavski VD, Klimov VV, Los DA, Carpentier R, Mohanty P (2008) Heat stress: an overview of molecular responses in photosynthesis. Photosynth Res 98:541-550

Anthoni U, Christophersen C, Hougaard L, Nielsen PH (1991) Quaternary ammonium compounds in the biosphere - an example of a versatile adaptive strategy. Comp Biochem Physiol B 99:1-18

Auton M, Rösgen J, Sinev M, Holthauzen LMF, Bolen DW (2011) Osmolyte effects on protein stability and solubility: a balancing act between backbone and side-chains. Biophys Chem 159:90-99

Baird AH, Marshall PA (2002) Mortality, growth and reproduction in scleractinian corals following bleaching on the Great Barrier Reef. Mar Ecol Prog Ser 237:133-141

Bennion BJ, Daggett V (2004) Counteraction of ureainduced protein denaturation by trimethylamine $\mathrm{N}$ oxide: a chemical chaperone at atomic resolution. Proc Natl Acad Sci USA 101:6433-6438

Blunden G, Smith BE, Irons MW, Yang M-H, Roch OG, Patel AV (1992) Betaines and tertiary sulphonium compounds from 62 species of marine algae. Biochem Syst Ecol 20: 373-388

* Brown BE, Downs CA, Dunne RP, Gibb SW (2002) Exploring the basis of thermotolerance in the reef coral Goniastrea aspera. Mar Ecol Prog Ser 242:119-129

* Bucher DJ, Harriott VJ, Roberts LG (1998) Skeletal microdensity, porosity and bulk density of acroporid corals. J Exp Mar Biol Ecol 228:117-138

Carlson BA (1999) Organism responses to rapid change: what aquaria tell us about nature. Am Zool 39:44-55

* Chen FCM, Benoiton NL (1976) A new method of quaternizing amines and its use in amino acid and peptide chemistry. Can J Chem 54:3310-3311

* Chen THH, Murata N (2011) Glycinebetaine protects plants against abiotic stress: mechanisms and biotechnological applications. Plant Cell Environ 34:1-20

de Vooys CGN, Geenevasen JAJ (2002) Biosynthesis and role in osmoregulation of glycine-betaine in the Mediterranean mussel Mytilus galloprovincialis LMK. Comp Biochem Physiol B 132:409-414

Edmunds PJ, Gates RD (2008) Acclimatization in tropical reef corals. Mar Ecol Prog Ser 361:307-310

Fan W, Zhang M, Zhang H, Zhang P (2012) Improved tolerance to various abiotic stresses in transgenic sweet potato (Ipomoea batatas) expressing spinach betaine aldehyde dehydrogenase. PLOS ONE 7:e37344

Fitt WK, Brown BE, Warner ME, Dunne RP (2001) Coral bleaching: interpretation of thermal tolerance limits and thermal thresholds in tropical corals. Coral Reefs 20: 51-65

Franklin DJ, Cedrés CMM, Hoegh-Guldberg O (2006) Increased mortality and photoinhibition in the symbiotic dinoflagellates of the Indo-Pacific coral Stylophora pistillata (Esper) after summer bleaching. Mar Biol 149: 633-642

Fukami H, Budd AF, Paulay G, Solé-Cava A, Chen CA, Iwao K, Knowlton N (2004) Conventional taxonomy obscures deep diverge between Pacific and Atlantic corals. Nature 427:832-835

* Gorbunov MY, Kolber ZS, Lesser MP, Falkowski PG (2001) Photosynthesis and photoprotection in symbiotic corals. Limnol Oceanogr 46:75-85

Guinn E, Pegram LM, Capp MW, Pollock MN, Record MT Jr (2011) Quantifying why urea is a protein denaturant, whereas glycine betaine is a protein stabilizer. Proc Natl Acad Sci USA 108:16932-16937

* Hawkins AJS, Klumpp DW (1995) Nutrition of the giant clam Tridacna gigas (L.). 2. Relative contributions of filter-feeding and the ammonium-nitrogen acquired and recycled by symbiotic algae towards total nitrogen requirements for tissue growth and metabolism. J Exp Mar Biol Ecol 190:263-290

*Hema R, Senthil-Kumar M, Shivakumar S, Reddy PC, Udayakumar M (2007) Chlamydomonas reinhardtii, a model system for functional validation of abiotic stress responsive genes. Planta 226:655-670

Hill R, Larkum AWD, Frankart C, Kühl M, Ralph PJ (2004) Loss of functional photosystem II reaction centres in zooxanthellae of corals exposed to bleaching conditions: using fluorescence rise kinetics. Photosynth Res 82: $59-72$

*Hill RW, Dacey JWH, Hill SD, Edward A, Hicks WA (2004) Dimethylsulfoniopropionate in six species of giant clams and the evolution of dimethylsulfide after death. Can J Fish Aquat Sci 61:758-764

* Hill RW, Li C, Jones AD, Gunn JP, Frade PR (2010) Abundant betaines in reef-building corals and ecological indicators of a photoprotective role. Coral Reefs 29:869-880

*Hoogenboom MO, Anthony KRN, Connolly SR (2006) Energetic cost of photoinhibition in corals. Mar Ecol Prog Ser 313:1-12

*Hughes TP, Baird AH, Bellwood DR, Card M and others (2003) Climate change, human impacts, and the resilience of coral reefs. Science 301:929-933

* Jeffrey SW, Haxo FT (1968) Photosynthetic pigments of symbiotic dinoflagellates (zooxanthellae) from corals and clams. Biol Bull 135:149-165

Jones RJ, Hoegh-Guldberg O (2001) Diurnal changes in the photochemical efficiency of the symbiotic dinoflagellates (Dinophyceae) of corals: photoprotection, photoinactivation and the relationship to coral bleaching. Plant Cell Environ 24:89-99

*Kasumyan AO, Døving KB (2003) Taste preferences in fishes. Fish Fish 4:289-347

*Keller MD, Kiene RP, Matrai PA, Bellows WK (1999) Production of glycine betaine and dimethylsulfoniopropionate in marine phytoplankton. I. Batch cultures. Mar Biol 135: $237-248$

KKlimov VV, Allakhverdiev SI, Nishiyama Y, Khorobrykh AA, Murata N (2003) Stabilization of the oxygen-evolving complex of photosystem II by bicarbonate and glycinebetaine in thylakoid and subthylakoid preparations. Funct Plant Biol 30:797-803

*Klumpp DW, Griffiths CL (1994) Contributions of phototrophic and heterotrophic nutrition to the metabolic and growth requirements of four species of giant clam (Tridacnidae). Mar Ecol Prog Ser 115:103-115

Lee SY, Jeong HJ, Kang NS, Jang TY, Jang SH, Lajeunesse TC (2015) Symbiodinium tridacnidorum sp. nov., a dinoflagellate common to Indo-Pacific giant clams, and a revised morphological description of Symbiodinium microadriaticum Freudenthal, emended Trench \& Blank. Eur J Phycol 50:155-172

* Leggat W, Buck BH, Grice A, Yellowlees D (2003) The impact of bleaching on the metabolic contribution of dinoflagellate symbionts to their giant clam host. Plant Cell Environ 26:1951-1961

Lesser MP (2011) Coral bleaching: causes and mechanisms. In: Dubinsky Z, Stambler N (eds) Coral reefs: an ecosys- 
tem in transition. Springer, New York, NY, p 405-419

Lesser MP, Farrell JH (2004) Exposure to solar radiation increases damage to both host tissues and algal symbionts of corals during thermal stress. Coral Reefs 23: 367-377

Li C, Hill RW, Jones AD (2010) Determination of betaine metabolites and dimethylsulfoniopropionate in coral tissues using liquid chromatography/time-of-flight mass spectrometry and stable isotope-labeled internal standards. J Chromatogr B 878:1809-1816

Li M, Li Z, Li S, Guo S, Meng Q, Li G, Yang X (2014) Genetic engineering of glycine betaine biosynthesis reduces heat-enhanced photoinhibition by enhancing antioxidative defense and alleviating lipid peroxidation in tomato. Plant Mol Biol Rep 32:42-51

McNeil SD, Nuccio ML, Hanson AD (1999) Betaines and related osmoprotectants. Targets for metabolic engineering of stress resistance. Plant Physiol 120:945-949

Middlebrook R, Hoegh-Guldberg O, Leggat W (2008) The effect of thermal history on the susceptibility of reefbuilding corals to thermal stress. J Exp Biol 211: 1050-1056

Moore RJ, Huxley CJ (1976) Aversive behaviour of crownof-thorns starfish to coral evoked by food-related chemicals. Nature 263:407-409

Naumann MS, Niggl W, Laforsch C, Glaser C, Wild C (2009) Coral surface area quantification - evaluation of established techniques by comparison with computer tomography. Coral Reefs 28:109-117

Papageorgiou GC, Murata N (1995) The unusually strong stabilizing effects of glycine betaine on the structure and function of the oxygen-evolving photosystem II complex. Photosynth Res 44:243-252

Perrino LA, Pierce SK (2000) Choline dehydrogenase kinetics contribute to glycine betaine regulation differences in Chesapeake Bay and Atlantic oysters. J Exp Zool 286: 250-261

Prasad KVSK, Saradhi PP (2004) Enhanced tolerance to photoinhibition in transgenic plants through targeting of glycinebetaine biosynthesis into the chloroplasts. Plant Sci 166:1197-1212

Rhodes D, Hanson AD (1993) Quaternary ammonium and tertiary sulfonium compounds in higher plants. Annu Rev Plant Physiol Plant Mol Biol 44:357-384

Roychoudhury A, Bieker A, Häussinger D, Oesterhelt F (2013) Membrane protein stability depends on the concentration of compatible solutes - a single molecule force spectroscopy study. Biol Chem 394:1465-1474

Sakamoto A, Murata N (2002) The role of glycine betaine in the protection of plants from stress: clues from transgenic plants. Plant Cell Environ 25:163-171

Schiller H, Dau H (2000) Preparation protocols for high-activ-

Editorial responsibility: Ronald Kiene,

Mobile, Alabama, USA ity photosystem II membrane particles of green algae and higher plants, $\mathrm{pH}$ dependence of oxygen evolution and comparison of the $\mathrm{S}_{2}$-state multiline signal by X-band EPR spectroscopy. J Photochem Photobiol B 55:138-144

Shirasawa K, Takabe T, Takabe T, Kishitani S (2006) Accumulation of glycinebetaine in rice plants that overexpress choline monooxygenase from spinach and evaluation of their tolerance to abiotic stress. Ann Bot 98: $565-571$

Street TO, Bolen DW, Rose GD (2006) A molecular mechanism for osmolyte-induced protein stability. Proc Natl Acad Sci USA 103:13997-14002

* Suenaga K (2004) Bioorganic studies on marine natural products with bioactivity, such as antitumor activity and feeding attractance. Bull Chem Soc Jpn 77:443-451

Takahashi S, Murata N (2008) How do environmental stresses accelerate photoinhibition? Trends Plant Sci 13: $178-182$

Turak E, DeVantier L (2005) Reef-building corals and coral communities of Pohnpei, Federated States of Micronesia: rapid ecological assessment of biodiversity and status. Conservation Society of Pohnpei, Kolonia

*Wang Y, Liu S, Zhang H, Zhao Y, Zhao H, Liu H (2014) Glycine betaine application in grain filling wheat plants alleviates heat and high light-induced photoinhibition by enhancing the $p s b A$ transcription and stomatal conductance. Acta Physiol Plant 36:2195-2202

* Warner ME, Fitt WK, Schmidt GW (1999) Damage to photosystem II in symbiotic dinoflagellates: a determinant of coral bleaching. Proc Natl Acad Sci USA 96:8007-8012

Weis VM (2008) Cellular mechanisms of cnidarian bleaching: stress causes the collapse of symbiosis. J Exp Biol 211:3059-3066

Winters G, Loya Y, Röttgers R, Beer S (2003) Photoinhibition in shallow-water colonies of the coral Stylophora pistillata as measured in situ. Limnol Oceanogr 48:1388-1393

* Yancey PH (2005) Organic osmolytes as compatible, metabolic and counteracting cytoprotectants in high osmolarity and other stresses. J Exp Biol 208:2819-2830

* Yancey PH, Heppenstall M, Ly S, Andrell RM, Gates RD, Carter VL, Hagedorn M (2010) Betaines and dimethylsulfoniopropionate as major osmolytes in cnidaria with endosymbiotic dinoflagellates. Physiol Biochem Zool 83: $167-173$

*Yang G, Rhodes D, Joly RJ (1996) Effects of high temperature on membrane stability and chlorophyll fluorescence in glycinebetaine-deficient and glycinebetaine-containing maize lines. Aust J Plant Physiol 23:437-443

* Yang X, Wen X, Gong H, Lu Q and others (2007) Genetic engineering of the biosynthesis of glycinebetaine enhances thermotolerance of photosystem II in tobacco plants. Planta 225:719-733

Submitted: February 14, 2017; Accepted: May 4, 2017

Proofs received from author(s): July 23, 2017 